\title{
New vector-like fermions and flavor physics
}

\author{
Koji Ishiwata, ${ }^{a}$ Zoltan Ligeti ${ }^{b}$ and Mark B. Wise ${ }^{c}$ \\ ${ }^{a}$ Institute for Theoretical Physics, Kanazawa University, \\ Kanazawa 920-1192, Japan \\ ${ }^{b}$ Ernest Orlando Lawrence Berkeley National Laboratory, University of California, \\ Berkeley, CA 94720, U.S.A. \\ ${ }^{c}$ Walter Burke Institute for Theoretical Physics, California Institute of Technology, \\ Pasadena, CA 91125, U.S.A. \\ E-mail: ishiwata@hep.s.kanazawa-u.ac.jp, ligeti@berkeley.edu, \\ wise@theory.caltech.edu
}

ABSTRACT: We study renormalizable extensions of the standard model that contain vectorlike fermions in a (single) complex representation of the standard model gauge group. There are 11 models where the vector-like fermions Yukawa couple to the standard model fermions via the Higgs field. These models do not introduce additional fine-tunings. They can lead to, and are constrained by, a number of different flavor-changing processes involving leptons and quarks, as well as direct searches. An interesting feature of the models with strongly interacting vector-like fermions is that constraints from neutral meson mixings (apart from $C P$ violation in $K^{0}-\bar{K}^{0}$ mixing) are not sensitive to higher scales than other flavor-changing neutral-current processes. We identify order $1 /(4 \pi M)^{2}$ (where $M$ is the vector-like fermion mass) one-loop contributions to the coefficients of the four-quark operators for meson mixing, that are not suppressed by standard model quark masses and/or mixing angles.

KEywords: Beyond Standard Model, Rare Decays, B-Physics, CP violation

ArXIV EPRINT: 1506.03484 


\section{Contents}

1 Introduction 1

2 The models 2

2.1 Lagrangians 3

2.2 New interactions with gauge bosons 5

3 Experimental constraints $\quad 8$

3.1 Leptonic models 8

3.2 Hadronic models 11

$\begin{array}{lll}3.2 .1 & \text { Meson decays involving a } \nu \bar{\nu} \text { pair } & 11\end{array}$

3.2.2 Meson decays to an $\ell^{+} \ell^{-}$pair 13

$\begin{array}{lll}3.2 .3 & \text { Semileptonic decays to } \ell^{+} \ell^{-} \text {pairs } & 15\end{array}$

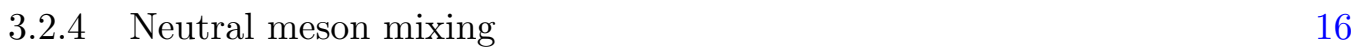

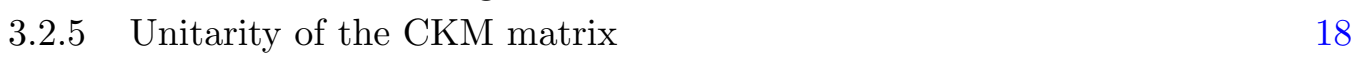

$\begin{array}{lll}4 & \text { Conclusions } & 19\end{array}$

$\begin{array}{ll}\text { A Diagonalizing matrix } & 21\end{array}$

B Numerical inputs and Wilson coefficients 22

\section{Introduction}

The driving force behind many of the extensions of the standard model (SM) has been the hierarchy puzzle. However, there may be reasons that the fine tuning of quadratically large contributions to the Higgs mass from very high momentum scales is acceptable (e.g., environmental reasons). This motivates the consideration of simple extensions of the standard model that do not address the hierarchy puzzle and do not introduce any additional fine tunings of parameters.

The SM provides no understanding why there are three generations of chiral fermions with masses that require weak symmetry breaking or why they only come in such simple representations of the gauge group. Vector-like fermions can be much heavier than the SM fermions, since their masses do not require weak symmetry breaking. For some choices of quantum numbers, such vector-like fermions can Yukawa couple to SM fermions. Given our lack of understanding of the number of generations and their quantum numbers, it seems worthwhile exploring the possibility that vector-like fermions exist. Of course, their masses may all be much larger than the weak scale, but it is not unreasonable that one such vector-like representation has a mass light enough that it can influence the next generation 
of flavor physics experiments. This motivates a study of extensions of the standard model with additional fermions that reside in a single vector-like representation of the SM gauge group with a mass $M$. (For some earlier studies of such models, see, e.g., refs. [1-10].) We focus on vector-like fermions that can have Yukawa couplings involving the Higgs field and the SM fermions. This allows them to influence flavor physics. There are several such models. These models have been studied in the literature previously, but here we consider all such models and compare the patterns of deviations from the SM they would give rise to. These models predict a very specific pattern for the contributions of beyond the standard model (BSM) physics to $\Delta F=1$ flavor-changing neutral-current (FCNC) processes compared to $\Delta F=2$ neutral meson mixings. For large masses $M$ the dominant order $1 /(4 \pi M)^{2}$ contribution to the coefficients of the four-quark operators responsible for neutral meson mixing arise at one loop, and are not suppressed by SM quark masses and/or weak mixing angles. We compute this contribution to meson mixing in the 7 models that involve strongly interacting vector-like fermions. In addition to considering $\Delta F=1$ and $\Delta F=2$ flavor-changing neutral-current processes, we also briefly discuss violations of lepton universality and unitarity of the CKM matrix that arises from the corrections to the $W$ boson couplings in these models.

The light neutrino masses play no role in our analysis so we take the SM to contain massless left-handed neutrinos. We are most interested in experiments that have reach in vector-like fermion mass of more than $\sim 10 \mathrm{TeV}$ when the Yukawa coupling constants of the vector-like fermions to the standard model fermions are around unity. For such masses, effects originating from the non-unitarity of the $3 \times 3$ CKM matrix can be neglected.

If the new fermion is in a real representation it can have a Majorana mass term which does not require a helicity partner in the spectrum. In that case, unless the Majorana mass is very large or the Yukawa coupling of the new neutral fermion to the SM neutrinos is very small, a SM neutrino gets an unacceptably large mass. Hence we do not consider models where the additional fermions come in a real representation of the gauge group. ${ }^{1}$

ATLAS and CMS have searched for strongly interacting vector-like fermions contained in several of the models discussed below [11-15]. The bounds from the Run 1 data constrain the masses of vector-like quarks typically at about the $M>800 \mathrm{GeV}$ level. These constraints come from the pair production of the vector-like fermions via their gauge couplings, and are (essentially) independent of the Yukawa couplings, which are the focus of this paper.

In section 2 we describe the models, section 3 explores the constraints from measurements both in the lepton and quark sectors, and section 4 contains our conclusions.

\section{The models}

There are 11 renormalizable models with vector-like fermions in complex representations of the standard model gauge group, where the vector-like fermions have renormalizable Yukawa couplings to the SM fermions through the Higgs doublet.

\footnotetext{
${ }^{1}$ There are two cases of this type. New fermions with SM quantum numbers $(1,1,0)$ and $(1,3,0)$.
} 
Models I and II have $\mathrm{SU}(2)_{L}$ singlet, $E$, and $\mathrm{SU}(2)_{L}$ triplet, $T_{e}$, vector-like fermions. Their $\mathrm{SU}(3)_{C} \times \mathrm{SU}(2)_{L} \times \mathrm{U}(1)_{Y}$ quantum numbers are $(1,1,-1)$ and $(1,3,-1)$. These vector-like fermions Yukawa couple through the Higgs field to the SM left-handed lepton doublets $L^{i}$.

Model III has vector-like fermions, $\ell$, with same gauge quantum numbers as the SM left-handed doublets, $(1,2,-1 / 2)$. They Yukawa couple to the SM right-handed charged lepton fields $e_{R}^{i}$. Model IV contains vector-like fermions, $l$, with $(1,2,-3 / 2)$ gauge quantum numbers, and they also Yukawa couple to $e_{R}^{i}$.

Models $\mathrm{V}$ and VI contain vector-like fermions $D$ and $U$ with the same quantum numbers as the SM right-handed down and up-type quarks, $(3,1,-1 / 3)$ and $(3,1,2 / 3)$. They Yukawa couple to the SM left-handed quark doublets $Q_{L}^{i}$. Models VII and VIII are similar with vector-like $\mathrm{SU}(2)_{L}$ triplet fermions $T_{d}$ and $T_{u}$ with gauge quantum numbers $(3,3,-1 / 3)$ and $(3,1,2 / 3)$ respectively.

There are three more models where the vector-like fermions Yukawa couple to the SM right-handed up-type, $u_{R}^{i}$ and down-type $d_{R}^{i}$ quarks. In Model IX the vector-like fermions $q_{u d}$ have the same quantum numbers as the SM left-handed quark doublets, $(3,2,1 / 6)$. In this case the new vector-like quark doublet couples to both $u_{R}^{i}$ and $d_{R}^{i}$. Model X has a vector-like quark doublet $q_{u}$ with quantum numbers $(3,2,7 / 6)$ that Yukawa couple through the Higgs doublet to the right-handed up-type quarks. Model XI has a vector-like quark doublet $q_{d}$ with quantum numbers $(3,2,-5 / 6)$ that Yukawa couples to the right-handed down-type quarks.

\subsection{Lagrangians}

The new terms in the Lagrange density which are added to the SM Lagrange density are:

$$
\begin{aligned}
\text { Model I }(1,1,-1): & \mathcal{L}_{\mathrm{BSM}}=\bar{E}(i \not D-M) E-\left(\lambda_{i} \bar{E}_{R} H^{\dagger} L_{L}^{i}+h . c .\right), \\
\text { Model II }(1,3,-1): & \mathcal{L}_{\mathrm{BSM}}=\operatorname{Tr}\left[\bar{T}_{e}(i \not D-M) T_{e}\right]-\left(\lambda_{i} H^{\dagger} \bar{T}_{e R} L_{L}^{i}+\text { h.c. }\right), \\
\text { Model III }(1,2,-1 / 2): & \mathcal{L}_{\mathrm{BSM}}=\bar{\ell}(i \not D-M) \ell-\left(\lambda_{i} \bar{e}_{R}^{i} H^{\dagger} \ell_{L}+\text { h.c. }\right), \\
\text { Model IV }(1,2,-3 / 2): & \mathcal{L}_{\mathrm{BSM}}=\bar{l}(i \not D-M) l-\left(\lambda_{i} \bar{e}_{R}^{i} H^{T} \epsilon l_{L}+\text { h.c. }\right), \\
\text { Model V }(3,1,-1 / 3): & \mathcal{L}_{\mathrm{BSM}}=\bar{D}(i \not D-M) D-\left(\lambda_{i} \bar{D}_{R} H^{\dagger} Q_{L}^{i}+\text { h.c. }\right), \\
\text { Model VI }(3,1,2 / 3): & \mathcal{L}_{\mathrm{BSM}}=\bar{U}(i \not D-M) U-\left(\lambda_{i} \bar{U}_{R} H^{T} \epsilon Q_{L}^{i}+\text { h.c. }\right), \\
\text { Model VII }(3,3,-1 / 3): & \mathcal{L}_{\mathrm{BSM}}=\operatorname{Tr}\left[\bar{T}_{d}(i \not D-M) T_{d}\right]-\left(\lambda_{i} H^{\dagger} \bar{T}_{d R} Q_{L}^{i}+\text { h.c. }\right), \\
\text { Model VIII }(3,3,2 / 3): & \mathcal{L}_{\mathrm{BSM}}=\operatorname{Tr}\left[\bar{T}_{u}(i \not D-M) T_{u}\right]-\left(\lambda_{i} H^{T} \epsilon \bar{T}_{u R} Q_{L}^{i}+\text { h.c. }\right), \\
\text { Model IX }(3,2,1 / 6): & \mathcal{L}_{\mathrm{BSM}}=\bar{q}_{u d}(i \not D-M) q_{u d} \\
& -\left(\lambda_{i}^{(u)} \bar{u}_{R}^{i} H^{T} \epsilon q_{u d L}+\lambda_{i}^{(d)} \bar{d}_{R}^{i} H^{\dagger} q_{u d L}+\text { h.c. }\right), \\
\text { Model X }(3,2,7 / 6): & \mathcal{L}_{\mathrm{BSM}}=\bar{q}_{u}(i \not D-M) q_{u}-\left(\lambda_{i} \bar{u}_{R}^{i} H^{\dagger} q_{u L}+\text { h.c. }\right), \\
\text { Model XI }(3,2,-5 / 6): & \mathcal{L}_{\mathrm{BSM}}=\bar{q}_{d}(i \not D-M) q_{d}-\left(\lambda_{i} \bar{d}_{R}^{i} H^{T} \epsilon q_{d L}+\text { h.c. }\right) .
\end{aligned}
$$


Here the $\mathrm{SU}(2)$ triplets $T_{e}, T_{u}$ and $T_{d}$ are represented by the two-by-two matrices

$$
\begin{array}{rlrl}
T_{e} & =\left(\begin{array}{cc}
T_{-1} / \sqrt{2} & T_{0} \\
T_{-2} & -T_{-1} / \sqrt{2}
\end{array}\right), & \bar{T}_{e} & =\left(\begin{array}{cc}
\bar{T}_{-1} / \sqrt{2} & \bar{T}_{-2} \\
\bar{T}_{0} & -\bar{T}_{-1} / \sqrt{2}
\end{array}\right), \\
T_{d}=\left(\begin{array}{cc}
T_{-1 / 3} / \sqrt{2} & T_{2 / 3} \\
T_{-4 / 3} & -T_{-1 / 3} / \sqrt{2}
\end{array}\right), & \bar{T}_{d}=\left(\begin{array}{cc}
\bar{T}_{-1 / 3} / \sqrt{2} & \bar{T}_{-4 / 3} \\
\bar{T}_{2 / 3} & -\bar{T}_{-1 / 3} / \sqrt{2}
\end{array}\right), \\
T_{u}=\left(\begin{array}{cc}
T_{2 / 3} / \sqrt{2} & T_{5 / 3} \\
T_{-1 / 3} & -T_{2 / 3} / \sqrt{2}
\end{array}\right), & \bar{T}_{u}=\left(\begin{array}{cc}
\bar{T}_{2 / 3} / \sqrt{2} & \bar{T}_{-1 / 3} \\
\bar{T}_{5 / 3} & -\bar{T}_{2 / 3} / \sqrt{2}
\end{array}\right),
\end{array}
$$

where the subscripts denote the charges of the fermions.

It is important to note that there are no new one-loop contributions to the ordinary lepton and quark mass matrices of the form

$$
m_{f_{i j}} \sim \frac{\lambda_{i}^{*} \lambda_{j} v}{16 \pi^{2}}
$$

where $v \simeq 174 \mathrm{GeV}$ is the Higgs vacuum expectation value. This is due to the approximate chiral symmetry, e.g., $e_{R}^{i} \rightarrow e^{i \alpha} e_{R}^{i}$ in Model I, which is only broken by the SM Yukawa couplings. This prevents such contributions to the light fermion mass matrix. So the $\lambda^{i}$ couplings can be order unity without the need for a cancellation in the SM fermion mass matrix.

The BSM effects caused by the vector-like fermions vanish as their mass $M \rightarrow \infty$. We are interested in very large $M$, greater than $10 \mathrm{TeV}$, and it is flavor-changing neutralcurrent processes that are sensitive to such BSM physics. In the SM, flavor-changing neutral currents are suppressed by small weak mixing angles and small SM quark or lepton masses. Furthermore, they do not occur at tree level. Since the neutrino masses are very small, charged lepton flavor violation in the SM is negligibly small. In the quark sector, despite their suppression, flavor-changing neutral-current processes that change flavor by one $\left(\Delta F=1\right.$, e.g., $\left.K^{+} \rightarrow \pi^{+} \nu \bar{\nu}\right)$ and by two $\left(\Delta F=2\right.$, e.g., $K^{0}-\bar{K}^{0}$ mixing $)$ have been observed.

In the limit where the SM Yukawa couplings vanish, the SM has a $\mathrm{U}(3)_{Q} \times \mathrm{U}(3)_{u_{R}} \times$ $\mathrm{U}(3)_{d_{R}} \times \mathrm{U}(3)_{L} \times \mathrm{U}(3)_{e_{R}}$ flavor symmetry. This symmetry forbids flavor-changing neutral currents. However in Models I-XI, even when the SM quark and lepton Yukawas are zero, the terms in the Lagrange density proportional to the Yukawa couplings $\lambda_{i}$ break that symmetry. That pattern of flavor symmetry breaking can be characterized using the spurion method. For example in Model V the BSM terms in the Lagrange density involving $\lambda_{i}$ are not invariant under the $\mathrm{U}(3)_{Q}$ transformations $Q_{L}^{i} \rightarrow V\left(Q_{L}\right)_{i j} Q_{L}^{j}$ (repeated flavor indices are summed). However, if the $\lambda_{i}$ also transform as $\lambda_{i} \rightarrow V\left(Q_{L}\right)_{i j}^{*} \lambda_{j}$, then the $\mathrm{U}(3)_{Q}$ symmetry is restored. This means that in Model V one-loop BSM physics associated with the high mass scale $M$ generates meson mixing through a term in the effective Lagrangian of the form,

$$
\mathcal{L}_{\text {meson }}^{(\mathrm{V})} \sim \frac{\lambda_{i}^{*} \lambda_{j} \lambda_{k}^{*} \lambda_{l}}{(4 \pi M)^{2}}\left(\bar{Q}_{L}^{i} \gamma_{\mu} Q_{L}^{j}\right)\left(\bar{Q}_{L}^{k} \gamma^{\mu} Q_{L}^{l}\right),
$$

and similarly for the other models. These corrections are not suppressed by small quark masses and/or mixing angles, and we have not found expressions for them in the literature. 
(We take the BSM Yukawa couplings $\lambda_{i}$ to be of order unity.) They are computed for Models V-XI in section 3.2.4. Corrections to meson mixing of order $\lambda_{i} \lambda_{j}^{*} /(4 \pi M)^{2}$ that are also suppressed by weak mixing angles and/or quark masses were considered for Model $\mathrm{V}$ in ref. [16].

\subsection{New interactions with gauge bosons}

The new BSM contribution to the $Z$ coupling and to the $W$ arise at tree level, after integrating out the heavy fermion(s). They can be obtained ether by calculating Feynman diagrams or by diagonalizing the $4 \times 4$ fermion mass matrices as was discussed in ref. [17]. Here we explicitly show how to obtain the BSM contributions to the $Z$ couplings for Model I. (The same method can be applied to the other models.)

After electroweak symmetry breaking, the mass matrix in charged lepton sector becomes,

$$
\mathcal{L}_{m^{\hat{e}}}=-\overline{\hat{e}}_{L}^{A} \mathcal{M}_{A B}^{\hat{e}} \hat{e}_{R}^{B}+\text { h.c. },
$$

where roman capital indices $A$ and $B$ go over $\{0,1,2,3\}$, $\hat{e}$ is defined by $\hat{e} \equiv\left(E, e^{1}, e^{2}, e^{3}\right)^{T}$, and

$$
\mathcal{M}^{\hat{e}}=\left(\begin{array}{cccc}
M & 0 & 0 & 0 \\
\lambda_{1}^{*} v & m_{e 1} & 0 & 0 \\
\lambda_{2}^{*} v & 0 & m_{e 2} & 0 \\
\lambda_{3}^{*} v & 0 & 0 & m_{e 3}
\end{array}\right) .
$$

Here we assumed, without loss of generality, that the charged lepton fields $e_{L}^{i}$ (i.e., the lower components of the doublets $L_{L}^{i}$ ) are eigenstates of the charged lepton mass matrix in the SM (i.e., in the $\lambda_{i} \rightarrow 0$ limit). This matrix is diagonalized by the $4 \times 4$ unitary transformations $V_{L, R}^{\hat{e}}$,

$$
\hat{e}_{L, R}^{\prime}=V_{L, R}^{\hat{e}} \hat{e}_{L, R},
$$

where the prime denotes a mass eigenstate field. So

$$
V_{L}^{\hat{e}} \mathcal{M}^{\hat{e}} V_{R}^{\hat{e} \dagger}=\mathcal{M}_{\mathrm{diag}}^{\hat{e}} .
$$

Up to corrections suppressed by $(v / M)^{2}$

$$
V_{L}^{\hat{e}}=\left(\begin{array}{cccc}
1 & \lambda_{1} v / M & \lambda_{2} v / M & \lambda_{3} v / M \\
-\lambda_{1}^{*} v / M & 1 & 0 & 0 \\
-\lambda_{2}^{*} v / M & 0 & 1 & 0 \\
-\lambda_{3}^{*} v / M & 0 & 0 & 1
\end{array}\right), \quad V_{R}^{\hat{e}}=1_{4 \times 4} .
$$

Consequently the masses of the charged leptons in the SM part of the Lagrangian are approximately equal to the charged lepton masses, and the vector mass parameter $M$ is approximately the heavy vector-like lepton mass.

The $Z$ boson coupling is,

$$
\mathcal{L}^{(Z)}=-g_{Z} Z_{\mu}\left[\frac{1}{2} \overline{\hat{e}}_{L}^{\prime A}\left(V_{L}^{\hat{e}}\right)_{A 0} \gamma^{\mu}\left(V_{L}^{\hat{e} \dagger}\right)_{0 B} \hat{e}_{L}^{B}+\ldots\right],
$$


where the ellipses denote terms not containing the matrix $V_{L}^{\hat{e}}$. Here $g_{Z}=\sqrt{g_{1}^{2}+g_{2}^{2}}$ and $g_{1,2}$ are the gauge couplings of $\mathrm{U}(1)_{Y}$ and $\mathrm{SU}(2)_{L}$, respectively. There is some ambiguity in how the terms are organized since $V_{L}^{\hat{e}}$ is unitary. We have written the $Z$ couplings involving $V_{L}^{\hat{e}}$ so that the order $(v / M)^{2}$ corrections to the SM $Z$ couplings to the charged leptons can be read off using the order $(v / M)$ terms in $V_{L}^{\hat{e}}$ that we have explicitly calculated. From this it follows (removing the hats and primes) that the BSM couplings of the $Z$ boson to the light mass eigenstate charged leptons are,

$$
\text { Model I : } \mathcal{L}_{\mathrm{BSM}}^{(Z)}=-\sum_{i, j}\left(\frac{\lambda_{i}^{*} \lambda_{j} m_{Z}^{2}}{g_{Z} M^{2}}\right) \bar{e}_{L}^{i} \gamma^{\mu} e_{L}^{j} Z_{\mu},
$$

with $m_{Z}$ being the $Z$ boson mass. For simplicity, hereafter we remove the primes used to specify the mass eigenstate fields.

In the same way, the new $Z$ couplings to the quarks and charged leptons are obtained for the other models. (The $4 \times 4$ unitary matrices are given in appendix A.) The results are

$$
\begin{aligned}
& \text { Model II : } \mathcal{L}_{\mathrm{BSM}}^{(Z)}=-\sum_{i, j}\left(\frac{\lambda_{i}^{*} \lambda_{j} m_{Z}^{2}}{2 g_{Z} M^{2}}\right) \bar{e}_{L}^{i} \gamma^{\mu} e_{L}^{j} Z_{\mu}, \\
& \text { Model III : } \mathcal{L}_{\mathrm{BSM}}^{(Z)}=\sum_{i, j}\left(\frac{\lambda_{i} \lambda_{j}^{*} m_{Z}^{2}}{g_{Z} M^{2}}\right) \bar{e}_{R}^{i} \gamma^{\mu} e_{R}^{j} Z_{\mu}, \\
& \text { Model IV : } \mathcal{L}_{\mathrm{BSM}}^{(Z)}=-\sum_{i, j}\left(\frac{\lambda_{i} \lambda_{j}^{*} m_{Z}^{2}}{g_{Z} M^{2}}\right) \bar{e}_{R}^{i} \gamma^{\mu} e_{R}^{j} Z_{\mu} .
\end{aligned}
$$

Note that in Models III and IV the BSM tree-level $Z$ couplings differ only by an overall sign.

Similarly for hadronic models,

$$
\begin{aligned}
& \text { Model V : } \mathcal{L}_{\mathrm{BSM}}^{(Z)}=-\sum_{i, j}\left(\frac{\lambda_{i}^{*} \lambda_{j} m_{Z}^{2}}{g_{Z} M^{2}}\right) \bar{d}_{L}^{i} \gamma^{\mu} d_{L}^{j} Z_{\mu} \\
& \text { Model VI : } \mathcal{L}_{\mathrm{BSM}}^{(Z)}=\sum_{i, j}\left(\frac{\lambda_{i}^{*} \lambda_{j} m_{Z}^{2}}{g_{Z} M^{2}}\right) \bar{u}_{L}^{i} \gamma^{\mu} u_{L}^{j} Z_{\mu} \\
& \text { Model IX : } \mathcal{L}_{\mathrm{BSM}}^{(Z)}=-\sum_{i, j}\left(\frac{\lambda_{i}^{(u)} \lambda_{j}^{(u) *} m_{Z}^{2}}{g_{Z} M^{2}} \bar{u}_{R}^{i} \gamma^{\mu} u_{R}^{j}-\frac{\lambda_{i}^{(d)} \lambda_{j}^{(d) *} m_{Z}^{2}}{g_{Z} M^{2}} \bar{d}_{R}^{i} \gamma^{\alpha} d_{R}^{j}\right) Z_{\mu}, \\
& \text { Model X : } \mathcal{L}_{\mathrm{BSM}}^{(Z)}=\sum_{i, j}\left(\frac{\lambda_{i} \lambda_{j}^{*} m_{Z}^{2}}{g_{Z} M^{2}}\right) \bar{u}_{R}^{i} \gamma^{\mu} u_{R}^{j} Z_{\mu}, \\
& \text { Model XI : } \mathcal{L}_{\mathrm{BSM}}^{(Z)}=-\sum_{i, j}\left(\frac{\lambda_{i} \lambda_{j}^{*} m_{Z}^{2}}{g_{Z} M^{2}}\right) \bar{d}_{R}^{i} \gamma^{\mu} d_{R}^{j} Z_{\mu} .
\end{aligned}
$$

So far, we have worked in the mass eigenstate basis most convenient for expressing the $Z$ couplings. For example, in Model $\mathrm{V}$ we have worked in a basis where in the $\lambda_{i} \rightarrow 0$ limit the down-type quarks in the Lagrangian are mass eigenstates, while in Model VI we have worked in the basis where the down-type quarks in the Lagrangian are mass eigenstate. In 
Model IX the couplings $\lambda^{(d)}$ correspond to the down quark mass eigenstate basis and the couplings $\lambda^{(u)}$ are in the up-quark mass eigenstate basis.

In case of vector-like triplets, i.e., Models VII and VIII, it is not possible to choose a basis where the CKM matrix, $V$, is absent from the $Z$ couplings. ${ }^{2}$ Choosing the up-type quarks in the Lagrange density to be mass eigenstates (in the $\lambda_{i} \rightarrow 0$ limit) implies for Model VII,

$$
\text { Model VII : } \mathcal{L}_{\mathrm{BSM}}^{(Z)}=-\sum_{i, j}\left(\frac{\lambda_{i}^{*} \lambda_{j} m_{Z}^{2}}{g_{Z} M^{2}}\right)\left(\bar{u}_{L}^{i} \gamma^{\mu} u_{L}^{j}+\frac{1}{2} \sum_{m, n} \bar{d}_{L}^{m} V_{m i}^{\dagger} \gamma^{\mu} V_{j n} d_{L}^{n}\right) Z_{\mu} .
$$

Similarly, in Model VIII the result is

$$
\text { Model VIII : } \mathcal{L}_{\mathrm{BSM}}^{(Z)}=\sum_{i, j}\left(\frac{\lambda_{i}^{*} \lambda_{j} m_{Z}^{2}}{g_{Z} M^{2}}\right)\left(\frac{1}{2} \bar{u}_{L}^{i} \gamma^{\mu} u_{L}^{j}+\sum_{m, n} \bar{d}_{L}^{m} V_{m i}^{\dagger} \gamma^{\mu} V_{j n} d_{L}^{n}\right) Z_{\mu} .
$$

We can transform these $Z$ couplings to the basis where the down-type quarks are mass eigenstates in the $\lambda_{i} \rightarrow 0$ limit by redefining the couplings $\lambda_{i} \rightarrow \sum_{k} \lambda_{k} V_{k i}^{\dagger}$. In this basis eqs. (2.32) and (2.33) become

$$
\text { Model VII : } \mathcal{L}_{\mathrm{BSM}}^{(Z)}=-\sum_{i, j}\left(\frac{\lambda_{i}^{*} \lambda_{j} m_{Z}^{2}}{g_{Z} M^{2}}\right)\left(\sum_{m, n} \bar{u}_{L}^{m} V_{m i} \gamma^{\mu} V_{j n}^{\dagger} u_{L}^{n}+\frac{1}{2} \bar{d}_{L}^{i} \gamma^{\mu} d_{L}^{j}\right) Z_{\mu},
$$

and

$$
\text { Model VIII : } \mathcal{L}_{\mathrm{BSM}}^{(Z)}=-\sum_{i, j}\left(\frac{\lambda_{i}^{*} \lambda_{j} m_{Z}^{2}}{g_{Z} M^{2}}\right)\left(\frac{1}{2} \sum_{m, n} \bar{u}_{L}^{m} V_{m i} \gamma^{\mu} V_{j n}^{\dagger} u_{L}^{n}+\bar{d}_{L}^{i} \gamma^{\mu} d_{L}^{j}\right) Z_{\mu} .
$$

There are corrections to the $W$ boson couplings as well. We write the couplings of the $W$-bosons to the left-handed light lepton and quark mass eigenstates in terms of $3 \times 3$ matrices $X$ and $Y$ as, ${ }^{3}$

$$
\mathcal{L}^{(W)}=-\frac{g_{2}}{\sqrt{2}} W_{\mu}^{+}\left[\bar{\nu}_{L}^{i} \gamma^{\mu} X_{i j} e_{L}^{j}\right]-\frac{g_{2}}{\sqrt{2}} W_{\mu}^{+}\left[\bar{u}_{L}^{i} \gamma^{\mu} Y_{i j} d_{L}^{j}\right]+\text { h.c. } .
$$

In the SM limit, $X$ is the inverse of the PMNS matrix and $Y$ is the CKM matrix. Because of the mixing with the vector-like leptons in the extensions of the SM discussed in this paper, $X$ and $Y$ are no longer unitary matrices. It is straightforward to express $X$ and $Y$ in terms of the components of the $4 \times 4$ diagonalization matrices $V_{L, R}$ in the various models.

In this paper we focus on violations of lepton universality and violations of unitarity of the CKM matrix. For that purpose we compute the quantities.

$$
\begin{aligned}
R_{k} & \equiv\left(X X^{\dagger}\right)_{k k}=\sum_{j=1,2,3} X_{k j} X_{k j}^{*}, \\
S_{l m} & \equiv\left(Y Y^{\dagger}\right)_{l m}=\sum_{j=1,2,3} Y_{l j} Y_{m j}^{*},
\end{aligned}
$$

\footnotetext{
${ }^{2}$ More precisely, the unitary matrix $V$ is the CKM matrix in the $\lambda_{i} \rightarrow 0$ limit.

${ }^{3}$ There are also right-handed currents in some of the models.
} 
at quadratic order in $\left(v^{2} / M^{2}\right)$, neglecting terms of higher order. We find in the models with vector-like leptons that

$$
\begin{aligned}
\text { Model I : } R_{k} & \simeq 1-\left|\lambda_{k}\right|^{2} \frac{v^{2}}{M^{2}}, \\
\text { Model II : } R_{k} & \simeq 1-\left(\frac{1}{2}\left|\lambda_{k}\right|^{2}+\left|\lambda_{k}^{\prime}\right|^{2}-\lambda_{k}^{*} \lambda_{k}^{\prime}-\lambda_{k} \lambda_{k}^{*}\right) \frac{v^{2}}{M^{2}}, \\
\text { Models III, IV : } R_{k} & \simeq 1 .
\end{aligned}
$$

In Model II we have worked in a basis where the charged leptons $\hat{e}^{j}$ are mass eigenstates in the SM part of the charged lepton mass matrix and so $\lambda_{j}^{\prime}=\sum_{i} \lambda_{i} U_{i j}$, where $U$ is the PMNS matrix.

In the models with vector-like quarks (neglecting the off diagonal elements of the CKM matrix)

$$
\begin{aligned}
& \text { Models V, VI : } S_{l m} \simeq \delta_{l m}-\lambda_{l}^{*} \lambda_{m} \frac{v^{2}}{M^{2}}, \\
& \text { Models VII, VIII : } \quad S_{l m} \simeq \delta_{l m}+\frac{1}{2} \lambda_{l}^{*} \lambda_{m} \frac{v^{2}}{M^{2}}, \\
& \text { Models IX, X, XI : } S_{l m} \simeq \delta_{l m} .
\end{aligned}
$$

At this order in the $v^{2} / M^{2}$ expansion, $T_{l m} \equiv\left(Y^{\dagger} Y\right)_{l m}=S_{l m}$.

\section{Experimental constraints}

For leptonic-extension models, i.e., Models I-IV, the constraints from $\mu \rightarrow e$ conversion, $\mu \rightarrow 3 e$ and $\tau \rightarrow 3 e$ are important. They are induced by the tree-level $Z$ couplings, which we derived in the previous section. On the other hand, for hadronic-extension models, i.e., Models V-XI, meson mixing, such as $K^{0}-\bar{K}^{0}$ and $D^{0}-\bar{D}^{0}$, are induced at one-loop level. In addition, $\Delta F=1$ FCNC processes, such as $K \rightarrow \pi \nu \bar{\nu}, K \rightarrow \mu^{+} \mu^{-}, B_{s} \rightarrow \ell^{+} \ell^{-}$, etc., are induced at tree level. We will derive effective Hamiltonians which are relevant for these processes and discuss the current experimental bounds, as well as future prospects. (For some recent studies of constraints on some of these models, see, e.g., refs. [18-20].)

For upper bounds on lepton flavor violating processes we quote bounds at 90\% CL, as do most experiments, whereas for other measurements we quote the $1 \sigma$ limits that are approximately $84 \%$ CL as one-sided bounds for Gaussian distributions.

\subsection{Leptonic models}

The flavor violating tree-level couplings in eqs. (2.23)-(2.26) give BSM tree-level contributions to the flavor-changing neural-current processes; for example, in the muon sector, to the $\mu \rightarrow 3 e$ rate and muon conversion to an electron in the presence of a nucleus. The amplitudes for these processes are both proportional to the same combination of parameters $\lambda_{1} \lambda_{2}^{*} / M^{2}$. The flavor diagonal terms in eqs. (2.23)-(2.26) give rise to violations of universality in lepton couplings to the $Z$. For typical couplings $\lambda$ these are not as sensitive 
to large values of $M$ as the flavor-changing charged lepton neutral currents are. Note that in these models the radiative decay $\mu \rightarrow e \gamma$ does not arise at tree level, only at one loop.

Let us first discuss $\mu \rightarrow e$ conversion in the presence of a nucleus. It is among the most powerful probes of charged lepton flavor violation beyond the standard model. The $\mu^{-}$ conversion rate to an $e^{-}$in the presence of a nucleus $N$ is usually quoted as a branching ratio normalized to the $\mathrm{SM}$ weak interaction, $\mu^{-} N \rightarrow \nu_{\mu} N^{\prime}$ capture rate. At present the most stringent bound is $\operatorname{Br}(\mu \rightarrow e$ conv. in $\mathrm{Au})<7 \times 10^{-13}$ at the $90 \%$ C.L. [21]. Future experiments will have a dramatically improved sensitivity to a branching ratio $\operatorname{Br}(\mu \rightarrow e$ conv. in $\mathrm{Al}) \sim 10^{-16}[22,23]$.

The reach, in mass scale for new physics, of the next generation charged lepton flavor violation (CLFV) experiments that search for $\mu$ to $e$ conversion on $\mathrm{Al}$ and for the radiative decay $\mu \rightarrow e \gamma$ was discussed in ref. [24]. They assume an effective Lagrangian of the form,

$$
\mathcal{L}_{\mathrm{CLFV}}=\frac{m_{\mu}}{(\kappa+1) \Lambda^{2}} \bar{\mu}_{R} \sigma_{\mu \nu} F^{\mu \nu} e_{L}+\frac{\kappa}{(\kappa+1) \Lambda^{2}} \bar{\mu}_{L} \gamma_{\mu} e_{L}\left(\bar{u}_{L} \gamma^{\mu} u_{L}+\bar{d}_{L} \gamma^{\mu} d_{L}\right)+\text { h.c. },
$$

and present the reach of these experiments in the $\kappa-\Lambda$ plane. For $\kappa \gg 1$ the limit on $\Lambda$ from conversion on gold is close to $1 \times 10^{3} \mathrm{TeV}$ while future planned experiments for conversion on aluminum are sensitive to $\Lambda \simeq 7.2 \times 10^{3} \mathrm{TeV}$. In Models I-IV the weak radiative decay proceeds at the one-loop level and so indeed $\kappa \gg 1$. Taking into account left-handed and right-handed quark currents which couple to $Z$ boson, the relations between $\Lambda$ and the vector-like lepton mass $M$ in Models $a=\mathrm{I}$, II, III, and IV are

$$
M^{(a)}=\Lambda\left\{\eta_{\mu \rightarrow e}^{(a)} \frac{\left|\lambda_{2} \lambda_{1}\right|}{3}\left[\left(2 \sin ^{2} \theta_{W}-1\right) \frac{Z}{A}+\frac{1}{2}\right]\right\}^{1 / 2},
$$

with $\eta_{\mu \rightarrow e}^{(\mathrm{I})}=2 \eta_{\mu \rightarrow e}^{(\mathrm{II})}=\eta_{\mu \rightarrow e}^{(\mathrm{III})}=\eta_{\mu \rightarrow e}^{(\mathrm{IV})}=1$ and $Z, A$ are atomic number, mass number of a nucleus, respectively. To obtain this expression we used the vector part in the quark current, neglecting the axial current. For Models III and IV, the right-handed lepton current occurs but it gives the same contribution to the rate as the left-handed one. On gold and aluminum eq. (3.2) implies that,

$$
M_{\mathrm{Au}}^{(\mathrm{a})}=0.31 \Lambda \sqrt{\eta_{\mu \rightarrow e}^{(a)}\left|\lambda_{2} \lambda_{1}\right|}, \quad M_{\mathrm{A} 1}^{(\mathrm{a})}=0.28 \Lambda \sqrt{\eta_{\mu \rightarrow e}^{(a)}\left|\lambda_{2} \lambda_{1}\right|},
$$

We obtain from figure 2 of ref. [24] that a future branching ratio limit $\operatorname{Br}(\mu \rightarrow e$ conv. in $\mathrm{Al})$ $<10^{-16}$ will imply $\Lambda>7.2 \times 10^{3} \mathrm{TeV}$ for large $\kappa$, yielding $M / \sqrt{\eta_{\mu \rightarrow e}^{(a)} \mid \lambda_{2} \lambda_{1}} \mid>2.0 \times 10^{3} \mathrm{TeV}$. The current limit on $\mu \rightarrow e$ conversion in Au implies that $\Lambda>9.5 \times 10^{2} \mathrm{TeV}$ [24], which according to eq. (3.3) implies the limits,

$$
M^{(a)}>\sqrt{\eta_{\mu \rightarrow e}^{(a)}\left|\lambda_{2} \lambda_{1}\right|} \times 2.9 \times 10^{2} \mathrm{TeV} .
$$

Charged lepton flavor violation is also constrained by the muon decay $\mu \rightarrow 3 e$ and similar $\tau$ decays. In the models $a=\{$ I, II, III, IV $\}$ the rates for these processes normalized to a leptonic weak decay mode are,

$$
\frac{\Gamma\left(e^{i} \rightarrow e^{j} e^{j} \bar{e}^{j}\right)}{\Gamma\left(e^{i} \rightarrow \nu^{i} e \bar{\nu}_{e}\right)}=\frac{\eta_{1}^{(a)}}{8 G_{F}^{2}} \frac{\left|\lambda_{i} \lambda_{j}\right|^{2}}{M^{4}},
$$


and

$$
\frac{\Gamma\left(e^{i} \rightarrow e^{j} e^{k} \bar{e}^{k}\right)}{\Gamma\left(e^{i} \rightarrow \nu^{i} e \bar{\nu}_{e}\right)}=\frac{\eta_{2}^{(a)}}{8 G_{F}^{2}} \frac{\left|\lambda_{i} \lambda_{j}\right|^{2}}{M^{4}} .
$$

For the decays where the leptons in the final state are all of the same type,

$$
\eta_{1}^{(\mathrm{I})}=4 \eta_{1}^{(\mathrm{II})}=2 \kappa_{L}^{2}+\kappa_{R}^{2}, \quad \eta_{1}^{(\mathrm{III})}=\kappa_{L}^{2}+2 \kappa_{R}^{2}
$$

where $\kappa_{L}=-1 / 2+\sin ^{2} \theta_{W}$ and $\kappa_{R}=\sin ^{2} \theta_{W}$, and $\theta_{W}$ is the Weinberg angle. Numerically: $\eta_{1}^{(\mathrm{I})} \simeq 0.20, \eta_{1}^{(\mathrm{II})} \simeq 0.049$ and $\eta_{1}^{(\mathrm{III})}=\eta_{1}^{(\mathrm{IV})} \simeq 0.18$. For the decays where two types of leptons occur in the final state,

$$
\eta_{2}^{(\mathrm{I})}=\eta_{2}^{(\mathrm{III})}=\eta_{2}^{(\mathrm{IV})}=\kappa_{L}^{2}+\kappa_{R}^{2}, \quad \eta_{2}^{(\mathrm{II})}=\frac{1}{4} \eta_{2}^{(\mathrm{I})} .
$$

Numerically; $\eta_{2}^{(\mathrm{I})}=\eta_{2}^{(\mathrm{III})}=\eta_{2}^{(\mathrm{IV})} \simeq 0.13$ and $\eta_{2}^{(\mathrm{II})} \simeq 0.031$. The $90 \%$ CL experimental limit, $\operatorname{Br}(\mu \rightarrow 3 e)<1.0 \times 10^{-12}[25]$ implies in Model I that $M / \sqrt{\left|\lambda_{1} \lambda_{2}\right|}>1.2 \times 10^{2} \mathrm{TeV}$. While the limits $\operatorname{Br}(\tau \rightarrow 3 \mu)<2.1 \times 10^{-8}$ and $\operatorname{Br}(\tau \rightarrow 3 e)<2.7 \times 10^{-8}$ [26] imply in Model I that $M / \sqrt{\left|\lambda_{2} \lambda_{3}\right|}>6.2 \mathrm{TeV}$ and $M / \sqrt{\left|\lambda_{1} \lambda_{3}\right|}>5.8 \mathrm{TeV}$. The limits on the rates for the $\tau$ decay channels $\tau \rightarrow \mu e \bar{e}$ and $\tau \rightarrow e \mu \bar{\mu}$ give slightly weaker limits because the $\eta_{2}$ 's are smaller than the $\eta_{1}$ 's.

The Mu3e experiment expects to reach in the absence of a signal the $90 \%$ CL limit, $\operatorname{Br}(\mu \rightarrow 3 e)<4 \times 10^{-16}[27]$, yielding in Model I, $M / \sqrt{\left|\lambda_{1} \lambda_{2}\right|}>8.2 \times 10^{2} \mathrm{TeV}$. The Belle II sensitivities for $\tau$ decays to three charged leptons are estimated at the few times $10^{-10}$ level, and several channels will give comparable sensitivity. The bounds $\operatorname{Br}(\tau \rightarrow 3 e)<4 \times 10^{-10}$ and $\operatorname{Br}(\tau \rightarrow 3 \mu)<4 \times 10^{-10}[28]$ would yield in Model I, $M / \sqrt{\left|\lambda_{1,2} \lambda_{3}\right|}>17 \mathrm{TeV}$.

Competitive constraints to the above processes also arise from the upper bounds on the $\tau \rightarrow e \pi, \tau \rightarrow \mu \pi, \tau \rightarrow e \rho$, and $\tau \rightarrow \mu \rho$ branching ratios. The vector-like fermions generate

$$
\Gamma\left(\tau \rightarrow e^{i} \pi\right)=\eta_{3}^{(a)} \frac{\left|\lambda_{3} \lambda_{i}\right|^{2}}{M^{4}} \frac{m_{\tau}^{3}}{256 \pi} f_{\pi}^{2},
$$

where $i=1,2, \eta_{3}^{(\mathrm{I})}=4 \eta_{3}^{(\mathrm{II})}=\eta_{3}^{(\mathrm{III})}=\eta_{3}^{(\mathrm{IV})}=1$, that is, $\eta_{3}^{(a)}=\eta_{2}^{(a)} /\left(\kappa_{L}^{2}+\kappa_{R}^{2}\right)$, and we neglected $m_{\pi}^{2} / m_{\tau}^{2}$. Similarly,

$$
\Gamma\left(\tau \rightarrow e^{i} \rho\right)=\eta_{3}^{(a)} \frac{\left|\lambda_{3} \lambda_{i}\right|^{2}}{M^{4}} \frac{\left(m_{\tau}^{2}-m_{\rho}^{2}\right)^{2}\left(m_{\tau}^{2}+2 m_{\rho}^{2}\right)}{256 \pi m_{\tau}^{3}}\left(1-2 \sin ^{2} \theta_{W}\right)^{2} f_{\rho}^{2} .
$$

Of the current 90\% CL experimental limits [29-31] the strongest bounds arise from $\operatorname{Br}(\tau \rightarrow$ $e \pi)<2.2 \times 10^{-8}$, which implies $M / \sqrt{\left|\lambda_{1} \lambda_{3}\right|}>7.0 \mathrm{TeV}$ in Model I, and $\operatorname{Br}(\tau \rightarrow \mu \rho)<$ $1.2 \times 10^{-8}$, which implies $M / \sqrt{\left|\lambda_{2} \lambda_{3}\right|}>7.4 \mathrm{TeV}$ in Model I. The bounds from $\tau \rightarrow e \rho$ and $\tau \rightarrow \mu \pi$ are only slightly weaker.

Concerning future sensitivity, the expected Belle II limits are [28],

$$
\begin{array}{ll}
\operatorname{Br}(\tau \rightarrow e \pi)<4.0 \times 10^{-10}, & \operatorname{Br}(\tau \rightarrow \mu \pi)<4.9 \times 10^{-10}, \\
\operatorname{Br}(\tau \rightarrow e \rho)<3.1 \times 10^{-10}, & \operatorname{Br}(\tau \rightarrow \mu \rho)<2.0 \times 10^{-10} .
\end{array}
$$


These imply that the strongest expected bounds in Model I will be $M / \sqrt{\left|\lambda_{1} \lambda_{3}\right|}>19 \mathrm{TeV}$ from $\tau \rightarrow e \pi$, and $M / \sqrt{\left|\lambda_{2} \lambda_{3}\right|}>21 \mathrm{TeV}$ from $\tau \rightarrow \mu \rho$. The expected sensitivities of the other channels are only slightly weaker, thus we can have high confidence in the experimental reach, but not in which channel will give the best bounds.

For generic BSM Yukawa couplings, $\lambda$, the reach for new physics is much greater for experiments that search for charged lepton flavor violation than those that seek violation of lepton universality. However, it is possible for non-generic $\lambda$ 's that the violations of universality are more important. We close this section by briefly commenting on the implications of the powerful constraint on $e-\mu$ charged current universality coming from pion decay $\pi^{+} \rightarrow e^{+} \bar{\nu}(\gamma), \mu^{+} \bar{\nu}(\gamma)$. The latest experimental result gives $g_{e} / g_{\mu}=0.9996 \pm$ 0.0012 [32] where $g_{e, \mu}$ are the charged-current couplings of $e$ and $\mu{ }^{4}$ In Models I-IV, $g_{e} / g_{\mu}=\sqrt{R_{1} / R_{2}}$, which implies, for example, in Model I that,

$$
M>\sqrt{\left.|| \lambda_{2}\right|^{2}-\left|\lambda_{1}\right|^{2} \mid} \times 4.4 \mathrm{TeV} .
$$

It should be possible to improve this bound by a factor of two in the future. Tau decay, on the other hand, gives constraints on other charged-current couplings, such as $g_{\tau} / g_{\mu}=$ $1.0011 \pm 0.0015$ [31], which yields, e.g., in Model I,

$$
M>\sqrt{\left.|| \lambda_{2}\right|^{2}-\left|\lambda_{3}\right|^{2} \mid} \times 6.2 \mathrm{TeV} .
$$

\subsection{Hadronic models}

To constrain Models V-XI, FCNC processes in the quark sector are most important. (For a recent study of $Z$-mediated FCNC effects, see, e.g., ref. [35].) We focus on leptonic and semileptonic decays and neutral meson mixing. In these models, constraints from nonleptonic decays are weaker.

\subsubsection{Meson decays involving a $\nu \bar{\nu}$ pair}

For kaon decays involving a neutrino-antineutrino pair, such as $K^{+} \rightarrow \pi^{+} \nu \bar{\nu}$, the effective Hamiltonian is

$$
\begin{aligned}
\mathcal{H} & =c_{\nu} \sum_{i}\left(\bar{s}_{L} \gamma_{\mu} d_{L}\right)\left(\bar{\nu}_{i L} \gamma^{\mu} \nu_{i L}\right)+c_{\nu}^{\prime} \sum_{i}\left(\bar{s}_{R} \gamma_{\mu} d_{R}\right)\left(\bar{\nu}_{i L} \gamma^{\mu} \nu_{i L}\right) \\
& =\frac{c_{\nu, V}}{2} \sum_{i}\left(\bar{s} \gamma_{\mu} d\right)\left(\bar{\nu}_{i L} \gamma^{\mu} \nu_{i L}\right)+\frac{c_{\nu, A}}{2} \sum_{i}\left(\bar{s} \gamma_{\mu} \gamma_{5} d\right)\left(\bar{\nu}_{i L} \gamma^{\mu} \nu_{i L}\right),
\end{aligned}
$$

where in the second line of eq. (3.14) the subscripts $V, A$ on the coefficients refer to the fact that these coefficients are for the vector and axial quark currents and $c_{\nu, V}=c_{\nu}^{\prime}+c_{\nu}$, $c_{\nu, A}=c_{\nu}^{\prime}-c_{\nu}$. (See appendix B.) Only the vector part of the quark current contributes to this process. In the SM $c_{\nu}^{\prime}=0$ and at NNLO, $\left|c_{\nu, V}^{(\mathrm{SM})}\right|=\left|c_{\nu}^{(\mathrm{SM})}\right| \simeq 1 /(89 \mathrm{TeV})^{2}$, with a few percent uncertainty [36-40]. The hadronic models modify $c_{\nu, V}$ additively. Obviously in this case it is convenient to work in the basis where the down-type quarks are mass eigenstates. ${ }^{5}$

\footnotetext{
${ }^{4}$ The constraint from Kaon decay [33, 34] is weaker.

${ }^{5}$ Going forward, for different processes we work in whatever basis makes the theoretical expressions simplest. This means that the $\lambda$ parameters are not always the same, but are linearly related through the CKM matrix.
} 
The tree-level $Z$ exchange BSM contributions to the coefficient $c_{\nu, V}$ in models $a=\{\mathrm{V}$, VII, VIII, IX, XI\} have magnitude

$$
\left|c_{\nu, V}^{(a)}\right|=\eta_{Z d}^{(a)} \frac{\left|\lambda_{1} \lambda_{2}\right|}{2 M^{2}}
$$

where for the FCNC down-type BSM contributions

$$
\eta_{Z d}^{(\mathrm{V})}=2 \eta_{Z d}^{(\mathrm{VII})}=\eta_{Z d}^{(\mathrm{VIII})}=\eta_{Z d}^{(\mathrm{IX})}=\eta_{Z d}^{(\mathrm{XI})}=1 .
$$

Note that for Model IX it is the $\lambda^{(d)}$ 's that occur in this expression. Models VI and X do not contribute to this process through tree-level $Z$ exchange.

The uncertainty of the SM prediction, $\mathrm{Br}^{(\mathrm{SM})}\left(K^{+} \rightarrow \pi^{+} \nu \bar{\nu}\right)=(7.8 \pm 0.8) \times 10^{-11}$, is dominated by that of $c_{\nu, V}^{(\mathrm{SM})}$, and not by its relation to the measured rate. Hence, the measurement $\operatorname{Br}\left(K^{+} \rightarrow \pi^{+} \nu \bar{\nu}\right)=(1.7 \pm 1.1) \times 10^{-10}[41]$ implies $0.8<\left|1+c_{\nu, V}^{(a)} / c_{\nu, V}^{(\mathrm{SM})}\right|^{2}<$ 3.6. ${ }^{6}$ In figure 1 the blue-shaded region shows the $1 \sigma$ allowed region in the parameter space of $M / \sqrt{\eta_{Z d}^{(a)}\left|\lambda_{1} \lambda_{2}\right|}$ and $\delta=\arg \left(c_{\nu, V}^{(a)} / c_{\nu, V}^{(\mathrm{SM})}\right)$. If the BSM contribution is aligned (constructive interference) with the SM, we find

$$
M^{(a)}>\sqrt{\eta_{Z d}^{(a)}\left|\lambda_{1} \lambda_{2}\right|} \times 66 \mathrm{TeV}
$$

This is not a bound in the strict sense. Anti-alignment, $\delta \sim \pi$, is possible for lower values of $M$. But it is more indicative of the new physics reach, since a larger region in $\delta$ is allowed for values of $M$ satisfying eq. (3.18).

The upcoming $10 \%$ measurement of $\operatorname{Br}\left(K^{+} \rightarrow \pi^{+} \nu \bar{\nu}\right)$, if consistent with the SM, would result in the $(1 \sigma)$ constraint $0.9<\left|1+c_{\nu, V}^{(a)} / c_{\nu, V}^{(\mathrm{SM})}\right|^{2}<1.1$, improving the sensitivity to BSM physics to about $2.8 \times 10^{2} \mathrm{TeV}$. This is shown as the red-shaded region in figure 1 . Again we emphasize, as is clear from figure 1, that quoting the weakest possible bound would not give the most useful impression of the scale sensitivity, as a solution around $c_{\nu, V}^{(a)}=-2 c_{\nu, V}^{(\mathrm{SM})}$ always remains, corresponding to a relatively low new physics scale. A $3 \sigma$ deviation from the SM is possible for $M^{(a)} / \sqrt{\eta_{Z d}^{(a)}\left|\lambda_{1} \lambda_{2}\right|}<1.7 \times 10^{2} \mathrm{TeV}$.

For the similar 3rd-2nd generation transition mediated by $b \rightarrow s \nu \bar{\nu}$, the SM prediction for the coefficient of the operator obtained from eq. (3.14) by $d \rightarrow s$ and $\bar{s} \rightarrow \bar{b}$ replacements is $\left|c_{\nu, V}^{(\mathrm{SM})}\right| \simeq 1 /(9.8 \mathrm{TeV})^{2}[42]$. This process has not been observed yet, and the best current bound, $\operatorname{Br}(B \rightarrow K \nu \bar{\nu})<1.6 \times 10^{-5}$, is about 4 times the SM prediction [43]. This yields for new physics aligned with the SM contribution, $M^{(a)}>\sqrt{\eta_{Z d}^{(a)}\left|\lambda_{2} \lambda_{3}\right|} \times 6.9 \mathrm{TeV}$. In case the new physics is anti-aligned, interfering destructively with the SM contribution, $M^{(a)}>\sqrt{\eta_{Z d}^{(a)}\left|\lambda_{2} \lambda_{3}\right|} \times 4.0 \mathrm{TeV}$. If the rate is at the SM level, Belle II expects to measure $\operatorname{Br}\left(B \rightarrow K^{*} \nu \bar{\nu}\right)$ with about $30 \%$ uncertainty $[28],{ }^{7}$ increasing the probed mass scales to about $19 \mathrm{TeV}$ (a $3 \sigma$ signal possible for $M^{(a)} / \sqrt{\eta_{Z d}^{(a)}\left|\lambda_{2} \lambda_{3}\right|}<11 \mathrm{TeV}$ ).

For $B \rightarrow \pi \nu \bar{\nu}$, Belle II expects to reach a sensitivity at the $1 \times 10^{-5}$ level [28], which will provide weaker bounds than $B \rightarrow \pi \ell^{+} \ell^{-}$in the models considered in this paper.

\footnotetext{
${ }^{6}$ Despite the stated uncertainty, the probability that all 7 observed events were due to background was quoted as 0.001 [41].

${ }^{7}$ At the SM level, $B \rightarrow K \nu \bar{\nu}$ is expected to get large backgrounds from $B \rightarrow K^{*} \nu \bar{\nu}$ [28]. While $\operatorname{Br}(B \rightarrow K \nu \bar{\nu})$ depends only on $C_{+\nu}, \operatorname{Br}\left(B \rightarrow K^{*} \nu \bar{\nu}\right)$ also depends on $C_{-\nu}$, slightly complicating the analysis.
} 


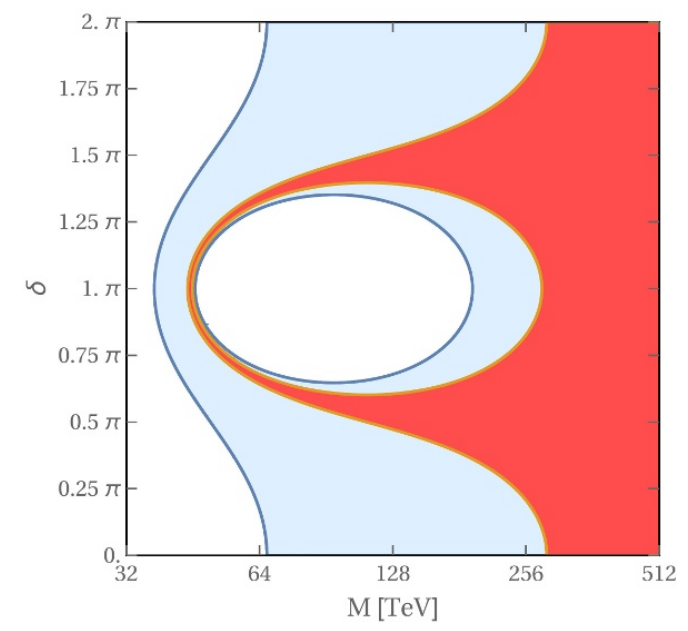

Figure 1. Constraints from $K^{+} \rightarrow \pi^{+} \nu \bar{\nu}$ on vector-like fermions in the $M / \sqrt{\eta_{Z d}^{(\mathrm{a})}\left|\lambda_{1} \lambda_{2}\right|}$ vs. $\delta=\arg \left(c_{\nu, V}^{(a)} / c_{\nu, V}^{(\mathrm{SM})}\right)$. The currently allowed $1 \sigma$ region is blue-shaded, corresponding to $0.8<$ $\left|1+c_{\nu, V}^{(a)} / c_{\nu, V}^{(\mathrm{SM})}\right|^{2}<3.6$, whereas a future $10 \%$ measurement in agreement with the SM, $0.9<$ $\left|1+c_{\nu, V}^{(a)} / c_{\nu, V}^{(\mathrm{SM})}\right|^{2}<1.1$, would constrain $M$ and $\delta$ to the red-shaded region.

\subsubsection{Meson decays to an $\ell^{+} \ell^{-}$pair}

The effective Hamiltonian differs from eq. (3.14) in that the coupling to the $\ell^{+} \ell^{-}$pair can either be left- or right-handed. We write the effective Hamiltonian as

$$
\mathcal{H}=\sum_{i=9,10}\left(c_{i} Q_{i}+c_{i}^{\prime} Q_{i}^{\prime}\right)
$$

where up to normalization the conventional choice of operator basis is

$$
\begin{aligned}
Q_{9} & =\left(\bar{b}_{L} \gamma_{\mu} s_{L}\right)\left(\bar{\ell} \gamma^{\mu} \ell\right), & Q_{9}^{\prime} & =\left(\bar{b}_{R} \gamma_{\mu} s_{R}\right)\left(\bar{\ell} \gamma^{\mu} \ell\right), \\
Q_{10} & =\left(\bar{b}_{L} \gamma_{\mu} s_{L}\right)\left(\bar{\ell} \gamma^{\mu} \gamma_{5} \ell\right), & Q_{10}^{\prime} & =\left(\bar{b}_{R} \gamma_{\mu} s_{R}\right)\left(\bar{\ell} \gamma^{\mu} \gamma_{5} \ell\right),
\end{aligned}
$$

with obvious replacements for $b \rightarrow d$ or $s \rightarrow d$ decays. We use the notation $c_{i}$ and $c_{i}^{\prime}$ to emphasize that these are dimensionful couplings, containing all terms multiplying the four-fermion operators $Q_{i}$ in eq. (3.20).

For $B_{s} \rightarrow \mu^{+} \mu^{-}$the SM gives $\left|c_{10}^{(\mathrm{SM})}\right| \simeq 1 /(17 \mathrm{TeV})^{2}$ and $\left|c_{10}^{\prime(\mathrm{SM})}\right| \simeq 0$. The amplitude for $B_{s} \rightarrow \mu^{+} \mu^{-}$is proportional to $c_{10, A}=c_{10}-c_{10}^{\prime}$. In the models with vector-like fermions the BSM contributions to the coefficients $c_{10, A}$ in models $a=\{\mathrm{V}, \mathrm{VII}, \mathrm{VIII}, \mathrm{IX}, \mathrm{XI}\}$ have magnitude

$$
\left|c_{10, A}^{(a)}\right|=\eta_{Z d}^{(a)} \frac{\left|\lambda_{2} \lambda_{3}\right|}{4 M^{2}}
$$

and the $\eta_{Z d}$ coefficients are the same as in eq. (3.17). The LHCb-CMS combination of their measurements, $\operatorname{Br}\left(B_{s} \rightarrow \mu^{+} \mu^{-}\right)=\left(2.8_{-0.6}^{+0.7}\right) \times 10^{-9}$, is quoted as the SM prediction times $0.76_{-0.18}^{+0.20}$ [44]. The $1 \sigma$ range of the measured $B_{s} \rightarrow \mu^{+} \mu^{-}$rate is slightly outside the SM and corresponds to the region, $0.96>\left|1+c_{10, A}^{(a)} / c_{10, A}^{(\mathrm{SM})}\right|^{2}>0.58$. The allowed one and two 


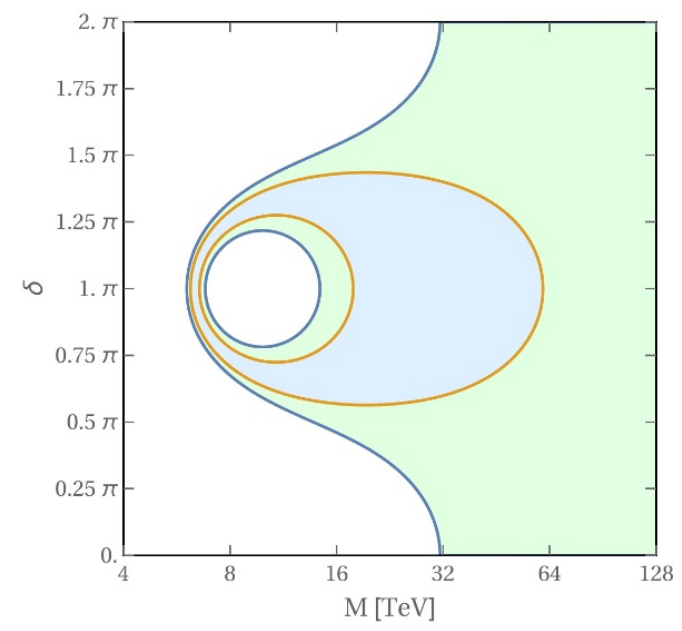

Figure 2. Constraints from $B_{s} \rightarrow \mu^{+} \mu^{-}$on vector-like fermions in the $M / \sqrt{\eta_{Z d}^{(\mathrm{a})}\left|\lambda_{2} \lambda_{3}\right|}$ vs. $\delta$ parameter space. The angle delta is defined in a similar way as in figure 1 . The blue-shaded region is allowed at $1 \sigma$ (indicating the mild tension with the SM), and the green-shaded region shows $2 \sigma$.

sigma regions of BSM parameter space are plotted in figure 2 using variables very similar to figure 1. If the BSM contribution is destructive (at $1 \sigma$ ),

$$
M^{(a)}>\sqrt{\eta_{Z d}^{(a)}\left|\lambda_{2} \lambda_{3}\right|} \times 18 \mathrm{TeV} .
$$

A future $10 \%$ measurement $[45,46]$ would increase this sensitivity to $\sim 40 \mathrm{TeV}$. Again we note that eq. (3.22) is not a true bound on $M$ but rather is meant to give a feeling for the reach in $M$ of current experimental data on this decay mode. There is a tuned region of BSM parameter space near $c_{10, A}^{(a)} / c_{10, A}^{(\mathrm{SM})}=-2$ that corresponds to a lower value of $M$.

For $B_{d} \rightarrow \mu^{+} \mu^{-}$the SM prediction is $\left|c_{10, A}^{(\mathrm{SM})}\right| \simeq 1 /(37 \mathrm{TeV})^{2}$. The LHCb-CMS combination, $\operatorname{Br}\left(B_{d} \rightarrow \mu^{+} \mu^{-}\right)=\left(3.9_{-1.4}^{+1.6}\right) \times 10^{-10}$ is quoted as the SM prediction times $3.7_{-1.4}^{+1.6}$ [44]. If the new contribution is constructive to the SM, then

$$
M^{(a)}>\sqrt{\eta_{Z d}^{(a)}\left|\lambda_{1} \lambda_{3}\right|} \times 16 \mathrm{TeV} .
$$

Accommodating the current central value would require $M^{(a)} / \sqrt{\eta_{Z d}^{(a)}\left|\lambda_{3} \lambda_{1}\right|} \simeq 19 \mathrm{TeV}$. In the HL-LHC era, this measurement will reach an uncertainty around $20 \%$ of the SM prediction $[45,46]$, increasing the mass reach to above $60 \mathrm{TeV}$.

The extraction of the short-distance part of the measured rate $\operatorname{Br}\left(K_{L} \rightarrow \mu^{+} \mu^{-}\right)=$ $(6.84 \pm 0.11) \times 10^{-9}[47]$ is subject to considerable uncertainties. The estimate $\operatorname{Br}\left(K_{L} \rightarrow\right.$ $\left.\mu^{+} \mu^{-}\right)_{\mathrm{SD}} \leq 2.5 \times 10^{-9}[48]$ is about 3 times the SM short-distance rate which follows from $\left|c_{10, A}^{\mathrm{SM}}\right|=1 /\left(1.8 \times 10^{2} \mathrm{TeV}\right)^{2}$. If the BSM component is aligned with the SM, we find

$$
M^{(a)}>\sqrt{\eta_{Z d}^{(a)} \mid \operatorname{Re}\left(\lambda_{1} \lambda_{2}^{*}\right)} \mid \times 100 \mathrm{TeV} .
$$

Unlike the bound from $K^{+} \rightarrow \pi^{+} \nu \bar{\nu}$, the prospect of improving this is not good, and the uncertainties are greater. 
Recently LHCb established a strong bound $\operatorname{Br}\left(D^{0} \rightarrow \mu^{+} \mu^{-}\right)<6.2 \times 10^{-9}$ at $90 \%$ CL [49], which is well above the SM level. In Models VI-X,

$$
\operatorname{Br}\left(D^{0} \rightarrow \mu^{+} \mu^{-}\right)=\tau_{D^{0}} \frac{\left|\lambda_{1} \lambda_{2}\right|^{2}}{M^{4}} \frac{\left[\eta_{Z u}^{(a)}\right]^{2}}{128 \pi} f_{D}^{2} m_{D} m_{\mu}^{2} \sqrt{1-4 m_{\mu}^{2} / m_{D}^{2}}
$$

where

$$
\eta_{Z u}^{(\mathrm{VI})}=\eta_{Z u}^{(\mathrm{VII})}=2 \eta_{Z u}^{(\mathrm{VIII})}=\eta_{Z u}^{(\mathrm{IX})}=\eta_{Z u}^{(\mathrm{X})}=1 .
$$

For Models VII and VIII we used the basis in eqs. (2.32) and (2.33). Using $f_{D}=$ $0.209 \mathrm{GeV}$ [50], we obtain

$$
M^{(a)}>\sqrt{\eta_{Z u}^{(a)}\left|\lambda_{1} \lambda_{2}\right|} \times 3.9 \mathrm{TeV} .
$$

It is possible that in the HL-LHC era the experimental bound will improve by a factor of $\sim 20$ [51].

\subsubsection{Semileptonic decays to $\ell^{+} \ell^{-}$pairs}

The bounds on $K_{L} \rightarrow \pi^{0} \ell^{+} \ell^{-}$are about an order of magnitude above the SM expectation, so the resulting constraints are weaker than those obtained from $K \rightarrow \pi \nu \bar{\nu}$ and $K_{L} \rightarrow$ $\mu^{+} \mu^{-}$.

LHCb recently measured $\operatorname{Br}\left(B^{+} \rightarrow \pi^{+} \mu^{+} \mu^{-}\right)=(2.3 \pm 0.6) \times 10^{-8}$ [52], consistent with the SM prediction quoted as $(2.0 \pm 0.2) \times 10^{-8}$. This was the first FCNC $b \rightarrow d$ decay observed (other than $B_{d}$ mixing). Requiring that the SM rate is not enhanced by more than $50 \%$ by new physics, using appendix B, we find

$$
M^{(a)}>\sqrt{\eta_{Z d}^{(a)}\left|\lambda_{1} \lambda_{3}\right|} \times 30 \mathrm{TeV}
$$

This bound is slightly stronger that that from $B_{d} \rightarrow \mu^{+} \mu^{-}$, moreover, the current $B^{+} \rightarrow$ $\pi^{+} \mu^{+} \mu^{-}$measurement only used $0.9 / \mathrm{fb}$ data. As the measurement gets more precise, a dedicated analysis of $B^{+} \rightarrow \pi^{+} \mu^{+} \mu^{-}$, possibly considering $\left[\mathrm{d} \Gamma\left(B^{+} \rightarrow \pi^{+} \mu^{+} \mu^{-}\right) / \mathrm{d} q^{2}\right] /[\mathrm{d} \Gamma(B \rightarrow$ $\left.\pi \ell \bar{\nu}) / \mathrm{d} q^{2}\right]$ to reduce theoretical uncertainties, is warranted.

The analysis of $B \rightarrow K \mu^{+} \mu^{-}$is very similar to $B^{+} \rightarrow \pi^{+} \mu^{+} \mu^{-}$, while $B \rightarrow K^{*} \mu^{+} \mu^{-}$is more complicated and does not give better bounds. Averaged over $\ell=e, \mu$, HFAG quotes $\operatorname{Br}\left(B \rightarrow K \ell^{+} \ell^{-}\right)=(4.8 \pm 0.4) \times 10^{-7}$ [31]. The experimental uncertainty is smaller than the theoretical one (due to the form factors). Demanding a less than $30 \%$ modification of the SM rate, we find $M^{(a)}>\sqrt{\eta_{Z d}^{(a)}\left|\lambda_{2} \lambda_{3}\right|} \times 17 \mathrm{TeV}$. This happens to be very close to the bound in eq. (3.22), but the prospects of improving that are better.

The inclusive decay rate $\operatorname{Br}\left(B \rightarrow X_{s} \ell^{+} \ell^{-}\right)=(5.0 \pm 0.6) \times 10^{-6}$ [31] depends in the models we consider on $\left|c_{9}\right|^{2}+\left|c_{9}^{\prime}\right|^{2}$ and $\left|c_{10}\right|^{2}+\left|c_{10}^{\prime}\right|^{2}$. In Models IX and XI, there is no interference between the SM and the new physics contributions, so the constraints are weak. We follow ref. [53], which studied the rates in the low- and high- $q^{2}$ regions and found that for the SM value of $c_{9}$ the constraint on $c_{10}$ in Models V, VII, and VIII is $0.88<1+c_{10}^{(a)} / c_{10}^{(\mathrm{SM})}<1.17($ at $1 \sigma)$. Here it is assumed that $c_{10}^{(a)} / c_{10}^{(\mathrm{SM})}$ is real. This implies

$$
M^{(a)}>\sqrt{\eta_{Z d}^{(a)}\left|\lambda_{2} \lambda_{3}\right|} \times 21 \mathrm{TeV} .
$$


In the up-quark sector, LHCb established a bound $\operatorname{Br}\left(D^{+} \rightarrow \pi^{+} \mu^{+} \mu^{-}\right)<7.3 \times 10^{-8}$ at 90\% CL [54], removing regions of $m_{\mu^{+} \mu^{-}}$with resonance contributions. We find that this bound is weaker on the models considered in this paper than the one from $\operatorname{Br}\left(D^{0} \rightarrow \mu^{+} \mu^{-}\right)$, and the latter is also theoretically cleaner.

For FCNC top decays, CMS has set the best bound so far, $\operatorname{Br}(t \rightarrow q Z)<5 \times 10^{-4}$ [55] at $95 \%$ CL (where $q=c, u$, corresponding to $i=2,1$ below, respectively). Comparing to the dominant $t \rightarrow b W$ rate, in models VI-X,

$$
\frac{\Gamma(t \rightarrow q Z)}{\Gamma(t \rightarrow b W)} \simeq \frac{2\left|\eta_{Z u}^{(a)} \lambda_{3} \lambda_{i}\right|^{2}}{g_{Z}^{4}} \frac{m_{Z}^{4}}{M^{4}}
$$

For Models VII and VIII we used again the basis in eqs. (2.32) and (2.33). We find $M^{(a)} / \sqrt{\eta_{Z u}^{(a)}\left|\lambda_{3} \lambda_{i}\right|}>0.96 \mathrm{TeV}$. The HL-LHC is expected to reach sensitivity at the $10^{-5}$ level [56], which will improve this bound to about $2.3 \mathrm{TeV}$. However, the direct (and $\lambda$-independent) searches are comparably sensitive, and are expected to remain to be so.

\subsubsection{Neutral meson mixing}

Since the new fermions interact with the Higgs field and the ordinary light quarks via Yukawa couplings, meson mixing, such as $K^{0}-\bar{K}^{0}, D^{0}-\bar{D}^{0}$, or $B^{0}-\bar{B}^{0}$, is induced. The effective Hamiltonian for these processes contains dimension-six four-quark operators with coefficients of mass dimension -2 . At tree level, through $Z$ exchange, the coefficients are of the form $\sim\left(\lambda_{i} \lambda_{j}^{*}\right)^{2} v^{2} / M^{4}$. However, coefficients of order $\left(\lambda_{i} \lambda_{j}^{*}\right)^{2} /(4 \pi M)^{2}$ are generated at one loop that are not CKM and/or quark-mass suppressed. For large $M$, these one-loop matching contributions are more important than tree-level $Z$ exchange. Furthermore, they are independent of the Higgs vacuum expectation value, $v$, and arise from short distances $\sim 1 / M$. They can be calculated in the symmetric phase and come from box diagrams with virtual scalars and the heavy vector-like fermions in the loop; see figure 3. The resulting effective Lagrangians are,

$$
\begin{aligned}
\mathcal{L}_{\text {meson }}^{(a)}= & -\eta_{\text {mix }}^{(a)} \frac{\left(\lambda_{i}^{*} \lambda_{j}\right)^{2}}{128 \pi^{2} M^{2}}\left[\sum_{k l m n}\left(\bar{u}_{L}^{k} V_{k i} \gamma_{\mu} V_{j l}^{\dagger} u_{L}^{l}\right)\left(\bar{u}_{L}^{m} V_{m i} \gamma_{\mu} V_{j n}^{\dagger} u_{L}^{n}\right)+\left(\bar{d}_{L}^{i} \gamma_{\mu} d_{L}^{j}\right)\left(\bar{d}_{L}^{i} \gamma^{\mu} d_{L}^{j}\right)\right] \\
& + \text { h.c., } \\
\mathcal{L}_{\text {meson }}^{(\mathrm{IX})}= & -\frac{\left(\lambda_{i}^{(u)} \lambda_{j}^{(u) *}\right)^{2}}{64 \pi^{2} M^{2}}\left(\bar{u}_{L}^{i} \gamma_{\mu} u_{L}^{j}\right)\left(\bar{u}_{L}^{i} \gamma^{\mu} u_{L}^{j}\right)+\frac{\left(\lambda_{i}^{(d)} \lambda_{j}^{(d) *}\right)^{2}}{64 \pi^{2} M^{2}}\left(\bar{d}_{L}^{i} \gamma_{\mu} d_{L}^{j}\right)\left(\bar{d}_{L}^{i} \gamma^{\mu} d_{L}^{j}\right)+h . c ., \\
\mathcal{L}_{\text {meson }}^{(\mathrm{X})}= & -\frac{\left(\lambda_{i} \lambda_{j}^{*}\right)^{2}}{128 \pi^{2} M^{2}}\left(\bar{u}_{R}^{i} \gamma_{\mu} u_{R}^{j}\right)\left(\bar{u}_{R}^{i} \gamma^{\mu} u_{R}^{j}\right)+h . c . \\
\mathcal{L}_{\text {meson }}^{\text {(XI) }}= & -\frac{\left(\lambda_{i} \lambda_{j}^{*}\right)^{2}}{128 \pi^{2} M^{2}}\left(\bar{d}_{R}^{i} \gamma_{\mu} d_{R}^{j}\right)\left(\bar{d}_{R}^{i} \gamma^{\mu} d_{R}^{j}\right)+h . c .,
\end{aligned}
$$

with $\eta_{\text {mix }}^{(\mathrm{V})}=\eta_{\text {mix }}^{(\mathrm{VI})}=1$ and $\eta_{\text {mix }}^{(\mathrm{VII})}=\eta_{\text {mix }}^{(\mathrm{VIII})}=5 / 4$. In the Lagrangians for Models V-VIII the down-type quarks are the SM mass eigenstates. For the remaining models the quark fields occurring both in their Lagrangians and in the effective Lagrangians for meson mixing can be taken to be SM mass eigenstates. 

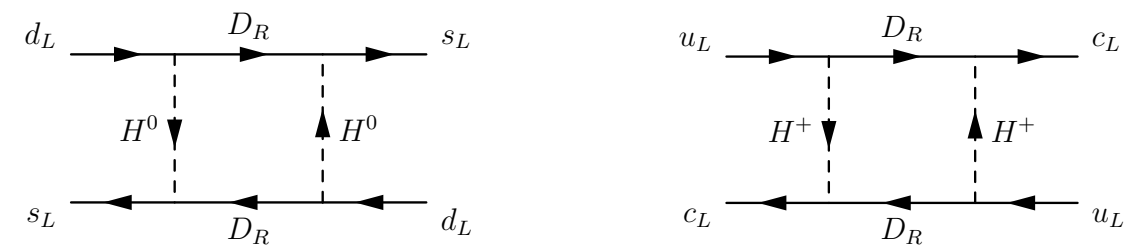

Figure 3. Diagrams contributing to $K$ and $D$ mixing in Model V.

The operators above are renormalized with the subtraction point at the scale $M$. They can be related through a QCD correction factor $\xi$ to scale invariant operators whose matrix elements can be evaluated using lattice QCD. So we write

$$
\left\langle\bar{P}\left|\left(\bar{q}_{(L, R)} \gamma^{\mu} q_{(L, R)}\right)^{2}\right| P\right\rangle=\frac{2}{3} \xi \hat{B}_{P} f_{P}^{2} m_{P}^{2},
$$

where $\hat{B}_{P}$ does not depend on subtraction point. For $K$ mixing, $f_{K}=156 \mathrm{MeV}$ and $\hat{B}_{K} \simeq 0.76$. In that case the QCD correction factor, $\xi$, is given in the leading logarithmic approximation [57] by

$$
\xi=\left[\frac{\alpha_{s}(M)}{\alpha_{s}\left(m_{t}\right)}\right]^{6 / 21}\left[\frac{\alpha_{s}\left(m_{t}\right)}{\alpha_{s}\left(m_{b}\right)}\right]^{6 / 23}\left[\frac{\alpha_{s}\left(m_{b}\right)}{\alpha_{s}\left(\mu_{0}\right)}\right]^{6 / 25}\left[\alpha_{s}\left(\mu_{0}\right)\right]^{6 / 25} \simeq\left[\alpha_{s}(M)\right]^{6 / 21} .
$$

The scale $\mu_{0}$ does not affect any physical results. For $10 \mathrm{TeV}<M<100 \mathrm{TeV}, \xi \simeq 0.5$, and this value is approximately the same for mixing in the $K, D$, and $B_{d, s}$ systems. We use the lattice QCD averages from ref. [50], and $\hat{B}_{D}=B_{D}^{\overline{\mathrm{MS}}}(3 \mathrm{GeV}) /\left[\alpha_{s}(3 \mathrm{GeV})\right]^{6 / 25} \simeq 1.0$ from a recent calculation of $B_{D}^{\overline{\mathrm{MS}}}(3 \mathrm{GeV})[58]$.

In the case of kaon mixing, we demand the new physics contribution to the real part of $M_{12}$ to be less than $40 \%$ of the experimental value of $\Delta m_{K}$ [59]. We take the $1 \sigma$ uncertainty on the determination of $\epsilon_{K}$ in the SM to be $25 \%$ (reading off the bound $h_{K}<0.35$ from ref. [60]). For all the (hadronic) models, except Model X, this results in the constraint

$$
M^{(a)}>\max \left[42 \sqrt{\eta_{\text {mix }}^{(a)}\left|\operatorname{Re}\left(\lambda_{2} \lambda_{1}^{*}\right)^{2}\right|}, 6.7 \times 10^{2} \sqrt{\eta_{\text {mix }}^{(a)}\left|\operatorname{Im}\left(\lambda_{2} \lambda_{1}^{*}\right)^{2}\right|}\right] \mathrm{TeV} .
$$

(Note that the $90 \%$ CL constraint from $\epsilon_{K}$ is only slightly weaker, replacing $6.7 \times 10^{2} \mathrm{TeV}$ by $6.3 \times 10^{2} \mathrm{TeV}$.) In the future, the $\epsilon_{K}$ constraint is expected to improve by about $\sqrt{2.4}$ [60], replacing $6.7 \times 10^{2} \mathrm{TeV}$ by $1.0 \times 10^{3} \mathrm{TeV}$ in eq. (3.37). Improvement in the real part is contingent upon lattice QCD calculations of the long-distance contributions to $\Delta m_{K}$; reaching $x \%$ precision would replace $42 \mathrm{TeV}$ in eq. (3.37) by $260 \mathrm{TeV} / \sqrt{x}$.

In $B_{d, s}$ meson mixing, the new physics contribution is conventionally parametrized as $M_{12}^{d, s}=\left(M_{12}^{d, s}\right)_{\mathrm{SM}} \times\left(1+h_{d, s} e^{2 i \sigma_{d, s}}\right)$. Until recently, the bounds on real $(2 \sigma=0, \bmod \pi$, that is MFV-like) and imaginary $(2 \sigma=\pi / 2, \bmod \pi)$ new physics contributions have been quite different [61,62]. This is no longer the case [60], and since we are most interested in physics reach, we simply quote the limits on the absolute values of the new physics contribution. In $B_{d, s}$ mixing, $h_{d}<0.3$ and $h_{s}<0.2$ [60] yield in all models except Model X, using $f_{B_{d}}=188 \mathrm{MeV}, f_{B_{s}}=226 \mathrm{MeV}, \hat{B}_{B_{d}}=1.27, \hat{B}_{B_{s}}=1.33[50]$,

$$
M^{(a)}>\sqrt{\eta_{\text {mix }}^{(a)}\left|\lambda_{3} \lambda_{1}\right|^{2}} \times 25 \mathrm{TeV}, \quad M^{(a)}>\sqrt{\eta_{\text {mix }}^{(a)}\left|\lambda_{3} \lambda_{2}\right|^{2}} \times 6.4 \mathrm{TeV} .
$$


(For the imaginary part in $B_{d}$ mixing, $25 \mathrm{TeV}$ should be replaced by $31 \mathrm{TeV}$, which is a smaller dependence on the phase of new physics than those ignored for $\Delta F=1 \mathrm{FCNC}$ transitions earlier.) In the above constraints $\eta_{\text {mix }}^{(\mathrm{IX})}=2, \eta_{\text {mix }}^{(\mathrm{XI})}=1$, and for Model IX it is the $\lambda^{(d)}$ 's that occur in the constraints. In the next decade these limits will improve to $h_{d}<0.05$ and $h_{s}<0.04$ [60], which will replace $25 \mathrm{TeV}$ by $61 \mathrm{TeV}$ and $6.4 \mathrm{TeV}$ by $14 \mathrm{TeV}$ in eq. (3.38).

The mixing of $D$ mesons is probably dominated by long-distance physics. Thus, we can only require that the new physics contribution does not exceed the measurement, i.e., $\Delta m_{D} / \Gamma_{D}<0.006$ [31]. (The significance of $\Delta m_{D} \neq 0$ is less than $2 \sigma$, so we use the upper bound of the $1 \sigma$ region. We do not distinguish between imaginary and real contributions to $M_{12}$ relative to the $\mathrm{SM}$; the bounds on the mass scale may differ by a factor $\sim 2$, depending on the value of $\Delta m_{D}$.) In contrast to above, the $\lambda^{(u)}$ couplings occur in the constraint for Model IX. Furthermore, in this case we choose the up-type quarks to be the SM mass eigenstate fields, so the factors of the CKM matrix move to the terms with the down-type quarks. Then $D$ mixing implies for all models except Model XI,

$$
M^{(a)}>\sqrt{\eta_{\text {mix }}^{(a)}\left|\lambda_{2} \lambda_{1}\right|^{2}} \times 48 \mathrm{TeV},
$$

where now $\eta_{\text {mix }}^{(\mathrm{X})}=1$. Note that for Models V-VIII the constraint from $D$ mixing is slightly stronger than from the real part of $K$ mixing. However, the constraints are actually a little different since the $\lambda$ 's in eqs. (3.38) and in (3.39) are not the same. They are linearly related through the CKM matrix. The future evolution of this bound is uncertain. While Belle II expects to measure $\Delta m_{D} / \Gamma_{D}$ with an uncertainty of 0.001 [28], the central value will matter for the bounds on new physics, and therefore we do not assume that this bound will improve.

\subsubsection{Unitarity of the CKM matrix}

The CKM matrix is not unitary in the extensions of the SM we are considering. The strongest constraints on violations of unitarity come form the first row and first column of the CKM matrix [47]

$$
S_{11}=0.9999 \pm 0.0006 \text { and } T_{11}=1.000 \pm 0.004 .
$$

In the models with vector-like quarks both of these constraints involve the same combination of couplings and we find

$$
M^{(a)}>\left|\lambda_{1}\right| \sqrt{\eta_{\mathrm{un}}^{(a)}} \times 7.8 \mathrm{TeV},
$$

where in $\eta_{\mathrm{un}}^{(\mathrm{V})}=\eta_{\mathrm{un}}^{(\mathrm{VI})}=2 \eta_{\mathrm{un}}^{(\mathrm{VII})}=2 \eta_{\mathrm{un}}^{(\mathrm{VIII})}=1$ and $\eta_{\mathrm{un}}^{(\mathrm{IX})}=\eta_{\mathrm{un}}^{(\mathrm{X})}=\eta_{\mathrm{un}}^{(\mathrm{XI})}=0$. For the second row we have the constraint

$$
S_{22}=1.002 \pm 0.027
$$

which yields

$$
M^{(a)}>\left|\lambda_{2}\right| \sqrt{\eta_{\text {un }}^{(a)}} \times 1.1 \mathrm{TeV}
$$




\section{Conclusions}

There are 11 renormalizable models that add to the SM vector-like fermions in a single (complex) representation of the gauge group that can Yukawa couple to the SM fermions through the Higgs field. These BSM fermions can have a mass $M$ that is much greater than the weak scale, since they have a mass term even in the absence of weak symmetry breaking. However, unlike BSM scalars, such fermions are technically natural. These models are a class of very simple extensions of the SM that do not worsen the SM hierarchy puzzle.

The masses of these vector-like fermions can take any value, up to the ultraviolet cutoff $\left(\sim M_{\mathrm{Pl}}\right)$, and so there is no particular reason that they should be in a region that can be probed experimentally. However, there are many such models and it is not unreasonable that one of them has vector-like fermions with masses in the experimentally testable range.

We considered the experimental constraints from flavor physics on the mass of these vector-like fermions to get a feel for the mass reach that the present experiments have for this class of models. We are primarily interested in very heavy vector-like fermions, say, with masses greater than $10 \mathrm{TeV}$. Hence it is flavor-changing neutral-current processes that provide the most important constraints. However, we also discussed violations of lepton universality and CKM matrix unitarity.

An important feature of these models is that for large $M$ the BSM contribution to meson mixing is either suppressed by a loop factor $\left(\sim 1 / 16 \pi^{2}\right)$ or a factor of $m_{Z}^{2} / M^{2}$ compared to processes that change flavor by one unit and are dominated by tree level through flavor-changing $Z$ exchange. This implies that, except for the case of the kaon $C P$ violation parameter $\epsilon_{K}$, the constraints on $M$ from meson mixing are not overwhelmingly strong. We computed the order $1 /(4 \pi M)^{2}$ one-loop contribution to the coefficients of the four-quark operators responsible for meson mixing that is not suppressed by SM quark masses or weak mixing angles.

We are interested in a rough assessment of the experimental reach. The strongest current bounds on the vector-like fermion masses and couplings in each of the 11 models studied in this paper are summarized in table 1 . We display bounds which are above or will get near the $10 \mathrm{TeV}$ level in the near future. Muon to electron conversion in a nucleus, $\mu \rightarrow 3 e$, the kaon $C P$-violating parameter $\epsilon_{K}$, and $K_{L} \rightarrow \mu^{+} \mu^{-}$are sensitive to vector-like fermion masses $\gtrsim 100 \mathrm{TeV}$ (for Yukawa couplings with magnitude unity). However, it is important to remember that the couplings $\lambda_{i}$ may have a flavor structure that suppresses these contributions relative to those involving the third generation.

We summarize the expected future sensitivity in table 2, where we only display sensitivities near or above $10 \mathrm{TeV}$. The Mu2e constraint will be improved dramatically in the next generation of experiments [22, 23]. The measured $K^{+} \rightarrow \pi^{+} \nu \bar{\nu}$ branching ratio corresponds to a mass reach around $70 \mathrm{TeV}$, which will increase substantially as the next generation experiments reach an uncertainty at about $10 \%$ of the SM rate, especially since the current central value is above the SM prediction (which has very small theoretical uncertainty). The improvement in the $\Delta m_{K}$ bound is entirely dependent on lattice QCD calculations, as discussed after eq. (3.37). The $B_{d, s}$ and $D$ mixing sensitivities will be improved by Belle II and LHCb. These experiments, and CMS and ATLAS, will also probe 


\begin{tabular}{|c|c|c|c|c|c|c|c|}
\hline \multirow{2}{*}{ Model } & Quantum & \multicolumn{6}{|c|}{ Present bounds on $M / \mathrm{TeV}$ and $\lambda_{i} \lambda_{j}$ for each $i j$ pair } \\
\hline & numbers & \multicolumn{2}{|c|}{$i j=12$} & \multicolumn{2}{|c|}{$i j=13$} & \multicolumn{2}{|c|}{$i j=23$} \\
\hline I & $(1,1,-1)$ & \multicolumn{2}{|c|}{$310^{a}$} & \multicolumn{2}{|c|}{$7.0^{b}$} & \multicolumn{2}{|c|}{$7.4^{c}$} \\
\hline II & $(1,3,-1)$ & \multicolumn{2}{|c|}{$220^{a}$} & \multicolumn{2}{|c|}{$4.9^{b}$} & \multicolumn{2}{|c|}{$5.2^{c}$} \\
\hline III & $(1,2,-1 / 2)$ & \multicolumn{2}{|c|}{$310^{a}$} & \multicolumn{2}{|c|}{$7.0^{b}$} & \multicolumn{2}{|c|}{$7.4^{c}$} \\
\hline IV & $(1,2,-3 / 2)$ & \multicolumn{2}{|c|}{$310^{a}$} & \multicolumn{2}{|c|}{$7.0^{b}$} & \multicolumn{2}{|c|}{$7.4^{c}$} \\
\hline & & $\Delta F=1$ & $\Delta F=2$ & $\Delta F=1$ & $\Delta F=2$ & $\Delta F=1$ & $\Delta F=2$ \\
\hline $\mathrm{V}$ & $(3,1,-1 / 3)$ & $66^{d}[100]^{e}$ & $\{42,670\}^{f}$ & $30^{g}$ & $25^{h}$ & $21^{i}$ & $6.4^{j}$ \\
\hline VI & $(3,1,2 / 3)$ & $3.9^{k}$ & $\{42,670\}^{f}$ & - & $25^{h}$ & - & $6.4^{j}$ \\
\hline VII & $(3,3,-1 / 3)$ & $47^{d}[71]^{e}$ & $\{47,750\}^{f}$ & $21^{g}$ & $28^{h}$ & $15^{i}$ & $7.2^{j}$ \\
\hline VIII & $(3,3,2 / 3)$ & $66^{d}[100]^{e}$ & $\{47,750\}^{f}$ & $30^{g}$ & $28^{h}$ & $21^{i}$ & $7.2^{j}$ \\
\hline $\operatorname{IX} \lambda^{(u)}$ & $\zeta_{2}, 1 / 6$ & $3.9^{k}$ & $67^{l}$ & - & $35^{h}$ & - & $9.1^{j}$ \\
\hline $\operatorname{IX} \lambda^{(d)}$ & $\int^{(3,2,1 / 0)}$ & $66^{d}[100]^{e}$ & $\{59,950\}^{f}$ & $30^{g}$ & $35^{h}$ & $18^{m}$ & $9.1^{j}$ \\
\hline $\mathrm{X}$ & $(3,2,7 / 6)$ & $3.9^{k}$ & $48^{l}$ & - & - & - & - \\
\hline XI & $(3,2-5 / 6)$ & $66^{d}[100]^{e}$ & $\{42,670\}^{f}$ & $30^{g}$ & $25^{h}$ & $18^{m}$ & $6.4^{j}$ \\
\hline
\end{tabular}

Table 1. Bounds from flavor-changing neutral currents on $M[\mathrm{TeV}] / \sqrt{\left|\lambda_{i} \lambda_{j}\right|}$ in the leptonic models, and from the $\Delta F=1$ constraints on the hadronic models. The $\Delta F=2$ bounds show $M / \sqrt{\left|\lambda_{i} \lambda_{j}\right|^{2}}$, except for $K$ meson mixing we show $\left\{M / \sqrt{\left|\operatorname{Re}\left(\lambda_{i} \lambda_{j}^{*}\right)^{2}\right|}, M / \sqrt{\left|\operatorname{Im}\left(\lambda_{i} \lambda_{j}^{*}\right)^{2}\right|}\right\}$. The strongest bounds arise from: a) $\mu$ to $e$ conversion; b) $\tau \rightarrow e \pi ; c) \tau \rightarrow \mu \rho$; d) $K \rightarrow \pi \nu \bar{\nu}$; e) $K_{L} \rightarrow \mu^{+} \mu^{-}$(this bound involves $\left.\left|\operatorname{Re}\left(\lambda_{1} \lambda_{2}^{*}\right)\right|\right)$; f) $K$ mixing; $\left.g\right) B \rightarrow \pi \mu^{+} \mu^{-}$; $\left.h\right) B_{d}$ mixing; i) $B \rightarrow X_{s} \ell^{+} \ell^{-}$; j) $B_{s}$ mixing; k) $D \rightarrow \mu^{+} \mu^{-}$; l) $D$ mixing; $\left.m\right) B_{s} \rightarrow \mu^{+} \mu^{-}$.

FCNC $B_{d, s}, D$, and $\tau$ decays much better than current bounds. The future sensitivities in table 2 correspond to estimated 50/ab Belle II [28] and 50/fb LHCb [45] sensitivities and CMS/ATLAS reach in rare decays on the same time scale. Compared to table 1, a greater number of the best bounds will come from purely leptonic rather than semileptonic decays. The sensitivities in table 2 may be realized in $\sim 10$ years.

\section{Acknowledgments}

We thank Doug Bryman, Tim Gershon, Yossi Nir, Karim Trabelsi, and Phill Urquijo for helpful comments. MBW thanks the Perimeter Institute for their hospitality during the completion of this work. ZL thanks the hospitality of the Aspen Center for Physics, supported by the NSF Grant No. PHY-1066293, during the completion of this work. ZL was supported in part by the Office of Science, Office of High Energy Physics, of the U.S. Department of Energy under contract DE-AC02-05CH11231. MBW was supported by the Gordon and Betty Moore Foundation through Grant No. 776 to the Caltech Moore Center for Theoretical Cosmology and Physics, and by the DOE Grant DE-SC0011632. He is also grateful for the support provided by the Walter Burke Institute for Theoretical Physics. 


\begin{tabular}{|c|c|c|c|c|c|c|c|}
\hline \multirow{2}{*}{ Model } & Quantum & \multicolumn{6}{|c|}{ Future bounds on $M / \mathrm{TeV}$ and $\lambda_{i} \lambda_{j}$ for each $i j$ pair } \\
\hline & numbers & \multicolumn{2}{|c|}{$i j=12$} & \multicolumn{2}{|c|}{$i j=13$} & \multicolumn{2}{|c|}{$i j=23$} \\
\hline I & $(1,1,-1)$ & \multicolumn{2}{|c|}{$2000^{a}$} & \multicolumn{2}{|c|}{$19^{b}$} & \multicolumn{2}{|c|}{$21^{c}$} \\
\hline II & $(1,3,-1)$ & \multicolumn{2}{|c|}{$1400^{a}$} & \multicolumn{2}{|c|}{$13^{b}$} & \multicolumn{2}{|c|}{$15^{c}$} \\
\hline III & $(1,2,-1 / 2)$ & \multicolumn{2}{|c|}{$2000^{a}$} & \multicolumn{2}{|c|}{$19^{b}$} & \multicolumn{2}{|c|}{$21^{c}$} \\
\hline IV & $(1,2,-3 / 2)$ & \multicolumn{2}{|c|}{$2000^{a}$} & \multicolumn{2}{|c|}{$19^{b}$} & \multicolumn{2}{|c|}{$21^{c}$} \\
\hline & & $\Delta F=1$ & $\Delta F=2$ & $\Delta F=1$ & $\Delta F=2$ & $\Delta F=1$ & $\Delta F=2$ \\
\hline $\mathrm{V}$ & $(3,1,-1 / 3)$ & $280^{d}$ & $\{100,1000\}^{e}$ & $60^{f}$ & $61^{g}$ & $39^{h}$ & $14^{i}$ \\
\hline VI & $(3,1,2 / 3)$ & $8.3^{j}$ & $\{100,1000\}^{e}$ & - & $61^{g}$ & - & $14^{i}$ \\
\hline VII & $(3,3,-1 / 3)$ & $200^{d}$ & $\{110,1100\}^{e}$ & $42^{f}$ & $68^{g}$ & $28^{h}$ & $16^{i}$ \\
\hline VIII & $(3,3,2 / 3)$ & $280^{d}$ & $\{110,1100\}^{e}$ & $60^{f}$ & $68^{g}$ & $39^{h}$ & $16^{i}$ \\
\hline $\operatorname{IX} \lambda^{(u)}$ & $(32) 1 / 6)$ & $8.3^{j}$ & $67^{k}$ & - & $86^{g}$ & - & $20^{i}$ \\
\hline $\operatorname{IX} \lambda^{(d)}$ & $(3,2,1 / 0)$ & $280^{d}$ & $\{140,1400\}^{e}$ & $60^{f}$ & $86^{g}$ & $39^{h}$ & $20^{i}$ \\
\hline $\mathrm{X}$ & $(3,2,7 / 6)$ & $8.3^{j}$ & $48^{k}$ & - & - & - & - \\
\hline $\mathrm{XI}$ & $(3,2-5 / 6)$ & $280^{d}$ & $\{100,1000\}^{e}$ & $60^{f}$ & $61^{g}$ & $39^{h}$ & $14^{i}$ \\
\hline
\end{tabular}

Table 2. Expected future bounds from flavor-changing neutral currents on $M[\mathrm{TeV}] / \sqrt{\left|\lambda_{i} \lambda_{j}\right|}$ in the leptonic models, and from the $\Delta F=1$ constraints on the hadronic models. The $\Delta F=2$ bounds show $M / \sqrt{\left|\lambda_{i} \lambda_{j}\right|^{2}}$, except for $K$ meson mixing we show $\left\{M / \sqrt{\left|\operatorname{Re}\left(\lambda_{i} \lambda_{j}^{*}\right)^{2}\right|}, M / \sqrt{\left|\operatorname{Im}\left(\lambda_{i} \lambda_{j}^{*}\right)^{2}\right|}\right.$. The bounds are from: $a) \mu$ to $e$ conversion; b) $\tau \rightarrow e \pi$; $) \tau \rightarrow \mu \rho$; $d) K \rightarrow \pi \nu \bar{\nu}$; $e$ ) $K$ mixing; f) $B_{d} \rightarrow \mu^{+} \mu^{-}$;g) $B_{d}$ mixing; $\left.h\right) B_{s} \rightarrow \mu^{+} \mu^{-}$; $\left.i\right) B_{s}$ mixing; $\left.j\right) D \rightarrow \mu^{+} \mu^{-}$; $k$ ) $D$ mixing.

\section{A Diagonalizing matrix}

Here we summarize the $4 \times 4$ diagonalizing matrices in the 11 models. Terms of order $(v / M)^{2}$ and higher are not explicitly displayed.

$$
\begin{aligned}
& \text { Model I : } \quad V_{L}^{\hat{e}}=\left(\begin{array}{cc}
1 & \lambda_{i} v / M \\
-\lambda_{i}^{*} v / M & 1_{3 \times 3}
\end{array}\right), \quad \quad V_{R}^{\hat{e}}=1_{4 \times 4}, \\
& \text { Model II : } \quad V_{L}^{\hat{e}}=\left(\begin{array}{cc}
1 & -\lambda_{i} v / \sqrt{2} M \\
\lambda_{i}^{*} v / \sqrt{2} M & 1_{3 \times 3}
\end{array}\right), \quad V_{R}^{\hat{e}}=1_{4 \times 4} \text {, } \\
& \text { Model III : } V_{L}^{\hat{e}}=1_{4 \times 4} \text {, } \\
& V_{R}^{\hat{e}}=\left(\begin{array}{cc}
1 & -\lambda_{i}^{*} v / M \\
\lambda_{i} v / M & 1_{3 \times 3}
\end{array}\right), \\
& \text { Model IV : } V_{L}^{\hat{e}}=1_{4 \times 4} \text {, } \\
& V_{R}^{\hat{e}}=\left(\begin{array}{cc}
1 & \lambda_{i}^{*} v / M \\
-\lambda_{i} v / M & 1_{3 \times 3}
\end{array}\right), \\
& \text { Model V : } V_{L}^{\hat{d}}=\left(\begin{array}{cc}
1 & \lambda_{i} v / M \\
-\lambda_{i}^{*} v / M & 1_{3 \times 3}
\end{array}\right), \quad V_{R}^{\hat{d}}=1_{4 \times 4}, \\
& \text { Model VI : } \quad V_{L}^{\hat{u}}=\left(\begin{array}{cc}
1 & -\lambda_{i} v / M \\
\lambda_{i}^{*} v / M & 1_{3 \times 3}
\end{array}\right), \quad V_{R}^{\hat{u}}=1_{4 \times 4} \text {, }
\end{aligned}
$$


Model VII : $\quad V_{L}^{\hat{u}}=\left(\begin{array}{cc}1 & \lambda_{i} v / M \\ -\lambda_{i}^{*} v / M & 1_{3 \times 3}\end{array}\right), \quad V_{R}^{\hat{u}}=1_{4 \times 4}$,

$$
V_{L}^{\hat{d}}=\left(\begin{array}{cc}
1 & -\lambda_{i} v / \sqrt{2} M \\
\lambda_{i}^{*} v / \sqrt{2} M & 1_{3 \times 3}
\end{array}\right), \quad V_{R}^{\hat{d}=1} 1_{4 \times 4},
$$

Model VIII : $\quad V_{L}^{\hat{u}}=\left(\begin{array}{cc}1 & -\lambda_{i} v / \sqrt{2} M \\ \lambda_{i}^{*} v / \sqrt{2} M & 1_{3 \times 3}\end{array}\right), \quad V_{R}^{\hat{u}}=1_{4 \times 4}$,

$$
V_{L}^{\hat{d}}=\left(\begin{array}{cc}
1 & -\lambda_{i} v / M \\
\lambda_{i}^{*} v / M & 1_{3 \times 3}
\end{array}\right), \quad V_{R}^{\hat{d}}=1_{4 \times 4},
$$

Model IX : $V_{L}^{\hat{u}}=1_{4 \times 4}$,

$$
V_{R}^{\hat{u}}=\left(\begin{array}{cc}
1 & \lambda_{i}^{(u) *} v / M \\
-\lambda_{i}^{(u)} v / M & 1_{3 \times 3}
\end{array}\right),
$$

$$
V_{L}^{\hat{d}}=1_{4 \times 4},
$$$$
V_{R}^{\hat{d}}=\left(\begin{array}{cc}
1 & -\lambda_{i}^{(d) *} v / M \\
\lambda_{i}^{(d)} v / M & 1_{3 \times 3}
\end{array}\right),
$$

Model X : $V_{L}^{\hat{u}}=1_{4 \times 4}$,

$$
V_{R}^{\hat{u}}=\left(\begin{array}{cc}
1 & -\lambda_{i}^{*} v / M \\
\lambda_{i} v / M & 1_{3 \times 3}
\end{array}\right) \text {, }
$$

Model XI : $\quad V_{L}^{\hat{d}}=1_{4 \times 4}$, $V_{R}^{\hat{d}}=\left(\begin{array}{cc}1 & -\lambda_{i}^{*} v / M \\ \lambda_{i} v / M & 1_{3 \times 3}\end{array}\right)$.

Finally, there are tree-level mass splittings among the heavy fermions, except for the $\mathrm{SU}(2)_{L}$ singlet models, due to electroweak symmetry breaking. The results are

$$
\begin{array}{rlrl}
\text { Model II : } & M_{0}=M+\Delta M, & M_{-1}=M+\Delta M / 2, \quad M_{-2}=M, \\
\text { Model III : } & M_{0}=M, & M_{-1}=M+\Delta M, \\
\text { Model IV : } & M_{-1}=M+\Delta M, & M_{-2}=M, \\
\text { Model VII : } & M_{2 / 3}=M+\Delta M, & M_{-1 / 3}=M+\Delta M / 2, \quad M_{-4 / 3}=M, \\
\text { Model VIII : } & M_{5 / 3}=M, & M_{2 / 3}=M+\Delta M / 2, \quad M_{-1 / 3}=M+\Delta M, \\
\text { Model IX : } & M_{2 / 3}=M-1 / 3=M+\Delta M, \\
\text { Model X : } & M_{5 / 3}=M, & M_{2 / 3}=M+\Delta M, \\
\text { Model XI : } & M_{-1 / 3}=M+\Delta M, & M_{-4 / 3}=M .
\end{array}
$$

In each model $\Delta M=\left(v^{2} / 2 M\right) \sum_{i}\left|\lambda_{i}\right|^{2}$.

\section{B Numerical inputs and Wilson coefficients}

We collect in this appendix expressions used to derive bounds on vector-like fermions from flavor-changing neutral-current processes, to facilitate easier comparison with and reproduction of our numerical results. Our goal in this paper is to study the sensitivities of many processes, so leading or next-to leading order results suffice. (For most processes the state of the art is one or two orders higher.) In many cases we ignore the SM uncertainties, when we know that they are subdominant effects. 
We adopt for the numerical values of the coupling constants $\alpha\left(m_{b}\right)=1 / 133, \sin ^{2} \theta_{W}=$ $0.23, g_{Z}=0.73$. For the top quark mass we use $\bar{m}_{t}\left(m_{t}\right)=165 \mathrm{GeV}$, obtained from the one-loop relation from the $m_{t}=173 \mathrm{GeV}$ (presumed) pole mass, extracted from fits to $t \bar{t}$ production at the Tevatron and the LHC.

The SM Wilson coefficients are as follows. For $K^{+} \rightarrow \pi^{+} \nu_{i} \bar{\nu}_{i}[36-40]$

$$
c_{+\nu}^{(\mathrm{SM})}=c_{\nu}^{(\mathrm{SM})}=\frac{\sqrt{2} G_{F} \alpha}{\pi \sin ^{2} \theta_{W}}\left[V_{t s}^{*} V_{t d} X\left(x_{t}\right)+V_{c s}^{*} V_{c d} \lambda^{4} P_{c}(X)\right]
$$

where $x_{t}=\bar{m}_{t}^{2} / m_{W}^{2}, \lambda=0.225, P_{c}(X) \simeq 0.4[38,40],{ }^{8}$ and

$$
X\left(x_{t}\right)=\frac{x_{t}}{8}\left[\frac{x_{t}+2}{x_{t}-1}+\frac{\left(3 x_{t}-6\right) \ln x_{t}}{\left(x_{t}-1\right)^{2}}\right]
$$

Here we have neglected the electroweak corrections.

For $B_{q} \rightarrow \mu^{+} \mu^{-}($where $q=s, d)$,

$$
c_{10}^{(\mathrm{SM})}=\frac{G_{F} \alpha}{\sqrt{2} \pi} V_{t b}^{*} V_{t q} \frac{Y\left(x_{t}\right)}{\sin ^{2} \theta_{W}}, \quad Y\left(x_{t}\right)=\frac{x_{t}}{8}\left(\frac{x_{t}-4}{x_{t}-1}+\frac{3 x_{t} \ln x_{t}}{\left(x_{t}-1\right)^{2}}\right) .
$$

(In the usual notation, $C_{10}=-Y\left(x_{t}\right) / \sin ^{2} \theta_{W} \simeq-4.2$.)

The short-distance contribution to the $K_{L} \rightarrow \mu^{+} \mu^{-}$rate can be written as [63]

$\Gamma\left(K_{L} \rightarrow \mu^{+} \mu^{-}\right)_{\mathrm{SD}}=\frac{G_{F}^{4} m_{W}^{4}}{4 \pi^{5}} \sin ^{4} \theta_{W} f_{K}^{2} m_{K} m_{\mu}^{2} \sqrt{1-\frac{4 m_{\mu}^{2}}{m_{K}^{2}}} \operatorname{Re}\left[\left(V_{t s}^{*} V_{t d} Y\left(x_{t}\right)+V_{c s}^{*} V_{c d} \lambda^{4} P_{c}\right)^{2}\right]$,

where $P_{c} \simeq 0.11$.

The $B \rightarrow K \mu^{+} \mu^{-}$rate is given by [42]

$$
\begin{aligned}
\frac{\mathrm{d} \Gamma\left(B \rightarrow K \mu^{+} \mu^{-}\right)}{\mathrm{d} q^{2}}= & \frac{G_{F}^{2} \alpha^{2} m_{B}^{3}}{1536 \pi^{5}}\left|V_{t b} V_{t s}\right|^{2}\left[\lambda_{K}\left(q^{2}\right)\right]^{3 / 2}\left\{f_{+}^{2}\left[\left|C_{9}^{\text {eff }}\left(q^{2}\right)\right|^{2}+\left|C_{10}+C_{10}^{\prime}\right|^{2}\right]\right. \\
& \left.+\frac{4 m_{b}^{2}}{\left(m_{B}+m_{K}\right)^{2}} f_{T}^{2}\left|C_{7}\right|^{2}+\frac{4 m_{b}}{m_{B}+m_{K}} f_{T} f_{+} \operatorname{Re}\left[C_{9}^{\text {eff }}\left(q^{2}\right) C_{7}^{*}\right]\right\},
\end{aligned}
$$

where $\lambda_{K}\left(q^{2}\right)$ is a phase space factor, $f_{+}$and $f_{T}$ are $q^{2}$-dependent form factors. The $B \rightarrow \pi \mu^{+} \mu^{-}$rate is obtained with obvious replacements. In the heavy quark limit $f_{+} / f_{T}=$ $1+\mathcal{O}\left(\Lambda_{\mathrm{QCD}} / m_{b}\right)$ [64], and model calculations are consistent with a mild $q^{2}$ dependence of this ratio. Hence, at the desired level of precision, the form factors can be pulled out of the $\{\ldots\}$ expression in eq. (B.5), obtaining simple approximations for the effect of new physics via $C_{10}$ and $C_{10}^{\prime}$. (This is impossible for $B \rightarrow K^{*} \mu^{+} \mu^{-}$, as $C_{7}$ enters with a $1 / q^{2}$ dependence in that case.) In the numerical analysis, we use $C_{7}=-0.33, C_{10}=-4.2$, and a mean value $\left|C_{9}^{\text {eff }}\left(q^{2}\right)\right|=4$.4. These estimates can be refined as the measurements improve.

\footnotetext{
${ }^{8}$ In our definition of $c_{\nu}^{(\mathrm{SM})}$ all lepton flavors (labeled by $i$ ) are included by using parameter $P_{c}(X)$, which is defined in $[36,37]$. Then $c_{\nu}^{(\mathrm{SM})}$ corresponds to the decay amplitude.
} 
Open Access. This article is distributed under the terms of the Creative Commons Attribution License (CC-BY 4.0), which permits any use, distribution and reproduction in any medium, provided the original author(s) and source are credited.

\section{References}

[1] G.C. Branco and L. Lavoura, On the Addition of Vector Like Quarks to the Standard Model, Nucl. Phys. B 278 (1986) 738 [InSPIRE].

[2] Y. Nir and D.J. Silverman, Z Mediated Flavor Changing Neutral Currents and Their Implications for CP Asymmetries in $B^{0}$ Decays, Phys. Rev. D 42 (1990) 1477 [InSPIRE].

[3] F. del Aguila, L. Ametller, G.L. Kane and J. Vidal, Vector Like Fermion and Standard Higgs Production at Hadron Colliders, Nucl. Phys. B 334 (1990) 1 [InSPIRE].

[4] G. Barenboim, F.J. Botella and O. Vives, Constraining models with vector-like fermions from FCNC in K and B physics, Nucl. Phys. B 613 (2001) 285 [hep-ph/0105306] [INSPIRE].

[5] G. Cacciapaglia, A. Deandrea, D. Harada and Y. Okada, Bounds and Decays of New Heavy Vector-like Top Partners, JHEP 11 (2010) 159 [arXiv:1007.2933] [INSPIRE].

[6] W. Altmannshofer, M. Bauer and M. Carena, Exotic Leptons: Higgs, Flavor and Collider Phenomenology, JHEP 01 (2014) 060 [arXiv:1308.1987] [INSPIRE].

[7] T. Ma, B. Zhang and G. Cacciapaglia, Triplet with a doubly-charged lepton at the LHC, Phys. Rev. D 89 (2014) 015020 [arXiv: 1309.7396] [INSPIRE].

[8] S.A.R. Ellis, R.M. Godbole, S. Gopalakrishna and J.D. Wells, Survey of vector-like fermion extensions of the Standard Model and their phenomenological implications, JHEP 09 (2014) 130 [arXiv: 1404.4398] [INSPIRE].

[9] F. del Aguila, M. Pérez-Victoria and J. Santiago, Observable contributions of new exotic quarks to quark mixing, JHEP 09 (2000) 011 [hep-ph/0007316] [INSPIRE].

[10] A.K. Alok, S. Banerjee, D. Kumar, S.U. Sankar and D. London, New-physics signals of a model with a vector-singlet up-type quark, Phys. Rev. D 92 (2015) 013002 [arXiv: 1504.00517] [INSPIRE].

[11] ATLAS collaboration, Search for vectorlike B quarks in events with one isolated lepton, missing transverse momentum and jets at $\sqrt{s}=8 \mathrm{TeV}$ with the ATLAS detector, Phys. Rev. D 91 (2015) 112011 [arXiv:1503.05425] [INSPIRE].

[12] ATLAS collaboration, A. Succurro Search for pair-produced vector-like quarks with the ATLAS detector, EPJ Web Conf. 60 (2013) 20037.

[13] CMS collaboration, Search for vector-like T quarks decaying to top quarks and Higgs bosons in the all-hadronic channel using jet substructure, JHEP 06 (2015) 080 [arXiv:1503.01952] [INSPIRE].

[14] CMS collaboration, Search for top-quark partners with charge $5 / 3$ in the same-sign dilepton final state, Phys. Rev. Lett. 112 (2014) 171801 [arXiv:1312.2391] [INSPIRE].

[15] CMS collaboration, Inclusive search for a vector-like $T$ quark with charge $\frac{2}{3}$ in pp collisions at $\sqrt{s}=8 \mathrm{TeV}$, Phys. Lett. B 729 (2014) 149 [arXiv:1311.7667] [InSPIRE].

[16] G. Barenboim and F.J. Botella, Delta $F=2$ effective Lagrangian in theories with vector-like fermions, Phys. Lett. B 433 (1998) 385 [hep-ph/9708209] [INSPIRE]. 
[17] K. Ishiwata and M.B. Wise, Phenomenology of heavy vectorlike leptons, Phys. Rev. D 88 (2013) 055009 [arXiv:1307.1112] [INSPIRE].

[18] F.J. Botella, G.C. Branco and M. Nebot, The Hunt for New Physics in the Flavour Sector with up vector-like quarks, JHEP 12 (2012) 040 [arXiv:1207.4440] [INSPIRE].

[19] S. Fajfer, A. Greljo, J.F. Kamenik and I. Mustac, Light Higgs and Vector-like Quarks without Prejudice, JHEP 07 (2013) 155 [arXiv: 1304.4219] [INSPIRE].

[20] G. Cacciapaglia, A. Deandrea, N. Gaur, D. Harada, Y. Okada and L. Panizzi, Interplay of vector-like top partner multiplets in a realistic mixing set-up, JHEP 09 (2015) 012 [arXiv: 1502.00370] [INSPIRE].

[21] SINDRUM II collaboration, W.H. Bertl et al., A search for muon to electron conversion in muonic gold, Eur. Phys. J. C 47 (2006) 337 [INSPIRE].

[22] Mu2E collaboration, R.J. Abrams et al., Mu2e Conceptual Design Report, arXiv:1211.7019 [INSPIRE].

[23] COMET collaboration, A. Kurup, The COherent Muon to Electron Transition (COMET) experiment, Nucl. Phys. Proc. Suppl. 218 (2011) 38 [INSPIRE].

[24] A. de Gouvêa and P. Vogel, Lepton Flavor and Number Conservation and Physics Beyond the Standard Model, Prog. Part. Nucl. Phys. 71 (2013) 75 [arXiv:1303.4097] [InSPIRE].

[25] SINDRUM collaboration, U. Bellgardt et al., Search for the Decay $\mu^{+} \rightarrow e^{+} e^{+} e^{-}$, Nucl. Phys. B 299 (1988) 1 [inSPIRE].

[26] K. Hayasaka et al., Search for Lepton Flavor Violating Tau Decays into Three Leptons with 719 Million Produced tau ${ }^{+}$tau ${ }^{-}$Pairs, Phys. Lett. B 687 (2010) 139 [arXiv:1001.3221] [INSPIRE].

[27] Mu3E collaboration, A. Bravar, The Muße Experiment at PSI, Nucl. Part. Phys. Proc. 260 (2015) 155 [INSPIRE].

[28] B. Golob, K. Trabelsi, P. Urquijo, Impact of Belle II on flavour physics, BELLE2-NOTE-0021, https://belle2.cc.kek.jp/ twiki/pub/B2TiP/WebHome/belle2-note-0021.pdf.

[29] Belle collaboration, Y. Miyazaki et al., Search for Lepton-Flavor-Violating tau Decays into Lepton and $f_{0}(980)$ Meson, Phys. Lett. B 672 (2009) 317 [arXiv:0810.3519] [INSPIRE].

[30] K. Hayasaka, Recent Tau Decay Results at B Factories: Lepton Flavor Violating Tau Decays, arXiv: 1010.3746 [INSPIRE].

[31] Heavy Flavor Averaging Group (HFAG) collaboration, Y. Amhis et al., Averages of b-hadron, c-hadron and $\tau$-lepton properties as of summer 2014, arXiv:1412.7515 [INSPIRE].

[32] PIENu collaboration, A. Aguilar-Arevalo et al., Improved Measurement of the $\pi \rightarrow e \nu$ Branching Ratio, Phys. Rev. Lett. 115 (2015) 071801 [arXiv:1506.05845] [INSPIRE].

[33] S. Ito et al., Status of the PIENU experiment at TRIUMF, J. Phys. Conf. Ser. 631 (2015) 012044 [INSPIRE].

[34] NA62 collaboration, C. Lazzeroni et al., Test of Lepton Flavour Universality in $K^{+} \rightarrow l^{+} \nu$ Decays, Phys. Lett. B 698 (2011) 105 [arXiv:1101.4805] [INSPIRE].

[35] A.J. Buras, F. De Fazio and J. Girrbach, The Anatomy of Z' and Z with Flavour Changing Neutral Currents in the Flavour Precision Era, JHEP 02 (2013) 116 [arXiv:1211.1896] [INSPIRE]. 
[36] G. Buchalla and A.J. Buras, The rare decays $K^{+} \rightarrow \pi^{+}$neutrino anti-neutrino and $K_{L} \rightarrow \mu^{+} \mu^{-}$beyond leading logarithms, Nucl. Phys. B 412 (1994) 106 [hep-ph/9308272] [INSPIRE].

[37] G. Buchalla and A.J. Buras, The rare decays $K \rightarrow \pi \nu \bar{\nu}, B \rightarrow X \nu \bar{\nu}$ and $B \rightarrow \ell^{+} \ell^{-}: A n$ update, Nucl. Phys. B 548 (1999) 309 [hep-ph/9901288] [InSPIRE].

[38] A.J. Buras, F. Schwab and S. Uhlig, Waiting for precise measurements of $K^{+} \rightarrow \pi^{+} \nu \bar{\nu}$ and $K_{L} \rightarrow \pi^{0} \nu \bar{\nu}$, Rev. Mod. Phys. 80 (2008) 965 [hep-ph/0405132] [INSPIRE].

[39] J. Brod and M. Gorbahn, Electroweak Corrections to the Charm Quark Contribution to $K+\rightarrow \pi+\nu \bar{\nu}$, Phys. Rev. D 78 (2008) 034006 [arXiv:0805.4119] [INSPIRE].

[40] A.J. Buras, D. Buttazzo, J. Girrbach-Noe and R. Knegjens, $K^{+} \rightarrow \pi^{+} \nu \bar{\nu}$ and $K_{L} \rightarrow \pi^{0} \nu \bar{\nu}$ in the Standard Model: Status and Perspectives, arXiv:1503.02693 [INSPIRE].

[41] E949 collaboration, A.V. Artamonov et al., New measurement of the $K^{+} \rightarrow \pi^{+} \nu \bar{\nu}$ branching ratio, Phys. Rev. Lett. 101 (2008) 191802 [arXiv:0808.2459] [INSPIRE].

[42] G. Buchalla, G. Hiller and G. Isidori, Phenomenology of nonstandard $Z$ couplings in exclusive semileptonic $b \rightarrow$ s transitions, Phys. Rev. D 63 (2000) 014015 [hep-ph/0006136] [INSPIRE].

[43] W. Altmannshofer, A.J. Buras, D.M. Straub and M. Wick, New strategies for New Physics search in $B \rightarrow K^{*} \nu \bar{\nu}, B \rightarrow K \nu \bar{\nu}$ and $B \rightarrow X_{s} \nu \bar{\nu}$ decays, JHEP 04 (2009) 022 [arXiv:0902.0160] [INSPIRE].

[44] LHCB, CMS collaboration, V. Khachatryan et al., Observation of the rare $B_{s}^{0} \rightarrow \mu^{+} \mu^{-}$ decay from the combined analysis of CMS and LHCb data, Nature $\mathbf{5 2 2}$ (2015) 68 [arXiv: 1411.4413] [INSPIRE].

[45] LHCb collaboration, Impact of the LHCb upgrade detector design choices on physics and trigger performance, LHCb-PUB-2014-040 (2014).

[46] M.O. Bettler, Rare decays of hadrons: experimental perspective, presentation at Physics at the High-Luminosity LHC, https://indico.cern.ch/event/360104/timetable/\#all.detailed.

[47] Particle Data Group collaboration, K.A. Olive et al., Review of Particle Physics, Chin. Phys. C 38 (2014) 090001 [inSPIRE].

[48] G. Isidori and R. Unterdorfer, On the short distance constraints from $K(L, S) \rightarrow \mu^{+} \mu^{-}$, JHEP 01 (2004) 009 [hep-ph/0311084] [INSPIRE].

[49] LHCb collaboration, Search for the rare decay $D^{0} \rightarrow \mu^{+} \mu^{-}$, Phys. Lett. B 725 (2013) 15 [arXiv: 1305.5059] [INSPIRE].

[50] S. Aoki et al., Review of lattice results concerning low-energy particle physics, Eur. Phys. J. C 74 (2014) 2890 [arXiv:1310.8555] [INSPIRE].

[51] T. Gershon, private communications.

[52] LHCb collaboration, First observation of the decay $B^{+} \rightarrow \pi^{+} \mu^{+} \mu^{-}$, JHEP 12 (2012) 125 [arXiv: 1210.2645] [INSPIRE].

[53] T. Huber, T. Hurth and E. Lunghi, Inclusive $\bar{B} \rightarrow X_{s} \ell^{+} \ell^{-}$: complete angular analysis and a thorough study of collinear photons, JHEP 06 (2015) 176 [arXiv:1503.04849] [INSPIRE].

[54] LHCb collaboration, Search for D+(s) to pi+ $\mu^{+} \mu^{-}$and $D_{(s)}^{+} \rightarrow \pi^{-} \mu^{+} \mu^{+}$decays, Phys. Lett. B 724 (2013) 203 [arXiv:1304.6365] [INSPIRE]. 
[55] CMS collaboration, Search for Flavor-Changing Neutral Currents in Top-Quark Decays $t \rightarrow Z q$ in pp Collisions at $\sqrt{s}=8$ TeV, Phys. Rev. Lett. 112 (2014) 171802 [arXiv: 1312.4194] [INSPIRE].

[56] ATLAS collaboration, Physics at a High-Luminosity LHC with ATLAS, arXiv:1307.7292 [INSPIRE].

[57] F.J. Gilman and M.B. Wise, Strong Interaction Corrections to $K^{0} \bar{K}^{0}$ Mixing in the Six Quark Model, Phys. Lett. B 93 (1980) 129 [INSPIRE].

[58] ETM collaboration, N. Carrasco et al., $\Delta S=2$ and $\Delta C=2$ bag parameters in the standard model and beyond from $N_{f}=2+1+1$ twisted-mass lattice QCD, Phys. Rev. D 92 (2015) 034516 [arXiv: 1505.06639] [INSPIRE].

[59] Z. Bai, N.H. Christ, T. Izubuchi, C.T. Sachrajda, A. Soni and J. Yu, $K_{L}-K_{S}$ Mass Difference from Lattice QCD, Phys. Rev. Lett. 113 (2014) 112003 [arXiv:1406.0916] [INSPIRE].

[60] J. Charles, S. Descotes-Genon, Z. Ligeti, S. Monteil, M. Papucci and K. Trabelsi, Future sensitivity to new physics in $B_{d}, B_{s}$ and $K$ mixings, Phys. Rev. D 89 (2014) 033016 [arXiv: 1309.2293] [INSPIRE].

[61] K. Blum, Y. Grossman, Y. Nir and G. Perez, Combining $K^{0}-\bar{K}^{0}$ mixing and $D^{0}-\bar{D}^{0}$ mixing to constrain the flavor structure of new physics, Phys. Rev. Lett. 102 (2009) 211802 [arXiv:0903.2118] [INSPIRE].

[62] G. Isidori, Y. Nir and G. Perez, Flavor Physics Constraints for Physics Beyond the Standard Model, Ann. Rev. Nucl. Part. Sci. 60 (2010) 355 [arXiv:1002.0900] [INSPIRE].

[63] A.J. Buras, F. De Fazio, J. Girrbach, R. Knegjens and M. Nagai, The Anatomy of Neutral Scalars with FCNCs in the Flavour Precision Era, JHEP 06 (2013) 111 [arXiv:1303.3723] [INSPIRE].

[64] N. Isgur and M.B. Wise, Relationship Between Form-factors in Semileptonic $\bar{B}$ and D Decays and Exclusive Rare $\bar{B}$ Meson Decays, Phys. Rev. D 42 (1990) 2388 [inSPIRE]. 